Bundesgesundheitsbl 2013 $\cdot 56: 1191-1215$

DOI 10.1007/s00103-013-1817-z

๑) Springer-Verlag Berlin Heidelberg 2013
Bekanntmachung des Bundesministeriums für Gesundheit

\section{Format für die Berichterstattung der zuständigen obersten Landesbehörden an das Bundesministerium für Gesundheit/Umweltbundesamt}

\author{
gemäß Richtlinie 98/83/EG und \\ Trinkwasserverordnung
}

\section{Einleitung}

Die rechtliche Grundlage des nationalen Berichtsformates bildet $\$ 21$ Absatz 2 der Trinkwasserverordnung (TrinkwV 2001). ${ }^{1,2}$ Diese Regelung bestimmt, dass die jährlichen Berichte der zuständigen obersten Landesbehörden über die Qualität des Trinkwassers dem von der EUKommission festgelegten Format einschließlich der dort genannten Mindestinformationen zu entsprechen haben. Das anzuwendende nationale Berichtsformat wird vom BMG nach Beteiligung der Länder mitgeteilt.

\footnotetext{
1 Trinkwasserverordnung in der Fassung der Bekanntmachung vom 28. November 2011 (BGBI. I S. 2370), die zuletzt durch Artikel 1 der Verordnung vom 5. Dezember 2012 (BGBI. I S. 2562) geändert worden ist.

2 Verwendete Abkürzungen: $\mathrm{BfG}=$ Bundesanstalt für Gewässerkunde; BMG = Bundesministerium für Gesundheit; BMU = Bundesministerium für Umwelt, Naturschutz und Reaktorsicherheit; EU = Europäische Union; ID = Identifikationsbezeichnung; NUTS = Nomenclature des unités territoriales statistiques (dt. Systematik der Gebietseinheiten für die Statistik); TWI = Trinkwasser-Installation (Hausinstallation); $\mathrm{TW}-\mathrm{RL}=$ Trinkwasserrichtlinie (Richtlinie 98/83/EG); WISE = Water Information System for Europe; UBA = Umweltbundesamt; $W V G=$ Wasserversorgungsgebiet(e).
}

2008 veröffentlichte das BMG in einer Mitteilung das „Format für die Berichterstattung der zuständigen Obersten Landesbehörden an das Bundesministerium für Gesundheit/Umweltbundesamt gemäß der Richtlinie 98/83/EG (Trinkwasserrichtlinie)“3 ${ }^{3}$ Die Mitteilung, der im Wesentlichen das „Guidance document on reporting under the Drinking Water Directive 98/83/EC“ (Leitfaden für die Berichterstattung gemäß Trinkwasserrichtlinie 98/83/EG) vom 8. Mai 2007 zugrunde lag, formulierte die nationalen Anforderungen an Berichterstattung und Informationsaustausch.

Die Wahrnehmung der Berichtspflichten nach TW-RL verlangte von Bund und Ländern, die Berichterstattung auf ein elektronisches Berichtswesen umzustellen. $\mathrm{Da}$ die hierzu erforderliche nationale Kommunikationsstruktur für die Behörden mit dem Berichtsportal „WasserBLIcK“ bereits vorhanden war und das BMU und die BfG anboten, diese Kommunikationswege für die Trinkwasserberichterstattung bereitzustellen und zu erweitern, richteten BMG, UBA und BfG eine zentrale Schnittstelle für Trinkwasserdaten bei BfG und UBA ein. Sie schufen damit die informationstechnischen

\footnotetext{
${ }^{3}$ Bundesgesundheitsbl. Gesundheitsforsch. Gesundheitsschutz 51, 2008:1078-1092.
}

Voraussetzungen, um die Trinkwasserdaten der Länder an den Bund (UBA/ BMG) nach Maßgabe des Formats für die Berichterstattung zu übermitteln. Die Landesbehörden passten die EDV-Systeme auf Länderebene den Bundesvorgaben an die Trinkwasserberichterstattung an und speisten ab dem Berichtsjahr 2009 bzw. 2010 die geforderten Daten in das Berichtsportal „WasserBLIcK“ ein. Auf diesem neuen, tabellenorientierten Format basiert der im Januar 2012 veröffentlichte Bericht des BMG und UBA an die Verbraucherinnen und Verbraucher über die Qualität von Wasser für den menschlichen Gebrauch für den Berichtszeitraum 2008 bis 2010.

\section{Berichterstattung gemäß TW-RL}

Die EU-Kommission gibt seit 1995 für WVG ab einer Größe von $1000 \mathrm{~m}^{3}$ pro Tag gelieferten Wassers für den menschlichen Gebrauch oder 5000 versorgten Personen ein Format für die Berichte nach TW-RL vor, das die Parameterwerte der TW-RL zugrunde legt. Nach TW-RL kann ein Mitgliedstaat zusätzliche Parameter und strengere Parameterwerte festsetzen, wenn es der Schutz der menschlichen Gesundheit im eigenen Hoheitsgebiet erfordert. So legt die TrinkwV 2001 
ergänzend für die Parameter Uran und Koloniezahl bei $36^{\circ} \mathrm{C}$ einen Grenzwert bzw. eine Anforderung fest und weicht bei Grenzwerten (z. B. Trihalogenmethane, Trübung) von den Parameterwerten der TW-RL ab. Die TrinkwV 2001 verpflichtet in den $\$ \$ 5$ bis 7 zur Einhaltung der Grenzwerte und Anforderungen, wie sie in Anlage 1 bis 3 TrinkwV 2001 festgeschrieben sind. Bei einer Nichteinhaltung eines Parameterwertes nach TW-RL liegt in jedem Fall auch eine Nichteinhaltung des Grenzwertes oder der Anforderung nach TrinkwV 2001 vor; umgekehrt kann ein solcher Rückschluss nicht vorgenommen werden (s. auch BMG-Mitteilung 2008, Vorwort).

\section{1 Änderungen des EU-Berichtsformates}

Im Februar 2011 vereinbarte der Komitologieausschuss nach Artikel 12 TW$\mathrm{RL}$, das „Guidance document on reporting under the Drinking Water Directive 98/83/EC" auf der Grundlage der in den Mitgliedstaaten damit gesammelten Erfahrungen zu überarbeiten, und betraute mit dieser Aufgabe eine Unterarbeitsgruppe, die im Oktober 2011 eine aktuelle Fassung des Leitfadens vorlegte. ${ }^{4}$ Die Änderungen richten sich u. a. auf die Probennahmestellen und -verfahren, auf vereinfachte und erweiterte Codes für Ursachen von Nichteinhaltung und Zeitrahmen für Abhilfemaßnahmen sowie auf eine modifizierte Darstellung von Untersuchungsergebnissen bei Nichteinhaltung von Parameterwerten im betreffenden WVG.

Die inhaltlichen Neuerungen im Format der Berichterstattung nach TW-RL verlangen, die Vorgaben zur Erfüllung der nationalen Informationspflichten daran anzupassen. Die Änderungen betreffen überwiegend die Tabellen des bisherigen Berichtsformates. Die Grundsätze 2-1 bis 2-11 in der oben zitierten BMG-Mitteilung bleiben hiervon unberührt und unterliegen ggf. der künftigen Fortschreibung der

\footnotetext{
${ }^{4}$ Das aktualisierte ${ }_{\text {Guidance document on }}$ reporting" vom Oktober 2011 liegt (bisher) nur in elektronischer Form und in englischer Sprache vor, s. unter: http://icm.eionet.europa.eu/schemas/dir199883ec/resources.
}

Schnittstelle durch BfG, BMG und UBA in Zusammenarbeit mit den zuständigen Landesbehörden.

Die obersten Landesbehörden berichten dem UBA ab dem Berichtsjahr 2014 nach dem hier vorgegebenen Format in der in $\$ 21$ Absatz 2 TrinkwV 2001 genannten Frist. Der Bericht ist als XMLDatei über die für Trinkwasser spezifizierte XML-Schnittstelle in die zentrale Datenhaltung des "WasserBLIcK“ bei der BfG einzustellen.

\subsection{Informationen, die dem UBA jährlich vorzulegen sind:}

\subsubsection{Informationen über die allgemeinen Rahmenbedingungen zur Trinkwasserversorgung im jeweiligen Land}

Das erforderliche Format ist durch - Tab. A1 bestimmt. ${ }^{5}$

\subsubsection{Informationen über Probennahmeverfahren, die bei der Überwachung der mikrobiologischen Parameter und der Metalle eingesetzt werden}

Diesbezügliche Angaben sind in

- Tab. A3b einzutragen.

\subsubsection{Kurzinformationen aus der Überwachung in Wasserversorgungsgebieten}

Diese Informationen sind nach dem Format der $\bullet$ Tab. A4 bereitzustellen.

\subsubsection{Informationen zur Trinkwasserqualität in Wasserversorgungsgebieten auf landesweiter Ebene}

Diese Informationen sind in dem Format gemäß $\bullet$ Tab. A5a wiederzugeben (Raum für weitere, auch fakultative Angaben zu Tab. A5a bietet $\bullet$ Tab. A5c).

\footnotetext{
5 Die Tabellennummerierung folgt dem Guidance document (s. Fußnote 4). Der hinzugefügte Großbuchstabe dient der besseren Zuordnung der im nationalen Format verwendeten Tabellen. Die Informationen, die das Guidance document in Tab. 2 und 3a einfordert, erteilen BMG und UBA.
}

\subsubsection{Informationen nach dem Format der 0 Tab. A5b über die produktspezifischen Parameter Acrylamid, Epichlorhydrin und Vinylchlorid}

\subsubsection{Information über die Ergebnisse der Nachforschungen bei Nichteinhaltung des Parameterwertes Clostridium perfringens \\ Dafür ist $\bullet$ Tab. A5d heranzuziehen.}

\subsubsection{Informationen zu Nichteinhaltungen von Parameterwerten in den unter 1.4.1 in Tab. A1 bezeichneten und betroffenen Wasserversorgungsgebieten}

Diese Informationen müssen den Vorgaben gemäß $\bullet$ Tab. A6 entsprechen. Hier sind auch jene von Artikel 9 TW-RL erfassten zugelassenen Abweichungen im Berichtsjahr zu berücksichtigen, über die das UBA bereits nach $\$ 10$ TrinkwV 2001 von den zuständigen obersten Landesbehörden unterrichtet worden ist (vgl. BMG-Mitteilung 2008, Grundsatz 2-11).

\subsection{Informationen über die Qualität von Wasser für den menschlichen Gebrauch an die Öffentlichkeit (s. auch BMG-Mitteilung 2008, Kapitel 4)}

Nach Artikel 13 Absatz 1 TW-RL sind in den Mitgliedstaaten Maßnahmen zu ergreifen, die sicherstellen, dass den Verbrauchern und Verbraucherinnen geeignetes und aktuelles Informationsmaterial über die Qualität von Wasser für den menschlichen Gebrauch zur Verfügung steht. Nach dem Format der $\bullet$ Tab. A11 ist zu dokumentieren, in welcher Form diese Informationen zur Trinkwasserbeschaffenheit der Öffentlichkeit verfügbar sind und wo ebenfalls die EU-Kommission solche Informationen abrufen bzw. erhalten kann. Gemäß dem Format von - Tab. A12 ist die Art der öffentlich zugänglichen Informationen nach Verwaltungsebene bzw. nach Größenordnung des Wasserversorgungsgebietes zu spezifizieren. 
Tab. A1 Allgemeine Informationen über die Rahmenbedingungen der Trinkwasserversorgung in dem Land. (Mit Ausnahme von Frage 1.2 werden nur Informationen über WVG gemeldet, in denen mehr als $1000 \mathrm{~m}^{3}$ Wasser für den menschlichen Gebrauch pro Tag verteilt oder mehr als 5000 Personen versorgt werden.)

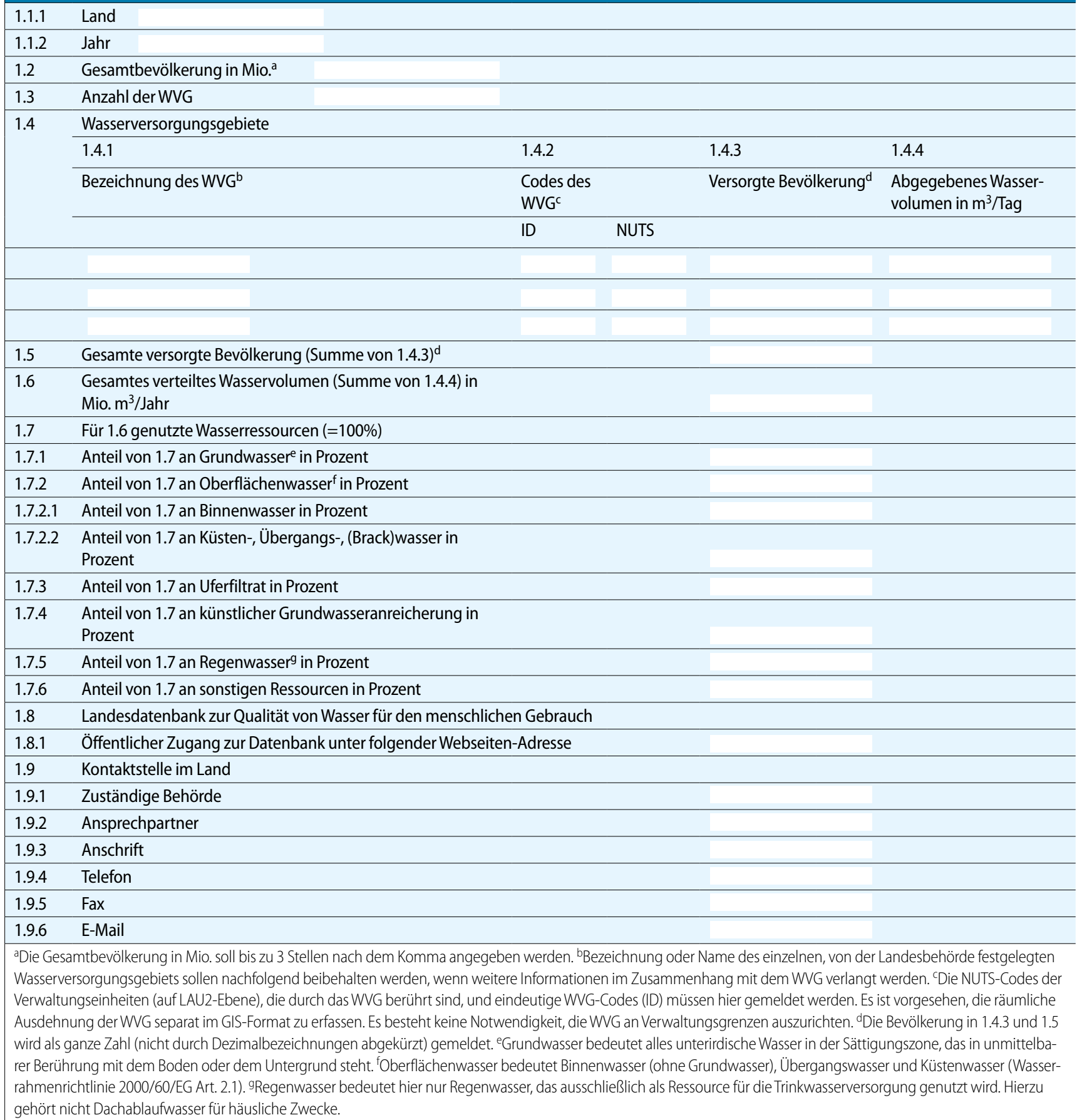




\section{Bekanntmachungen - Amtliche Mitteilungen}

Tab. A3b Probennahmeverfahren, die bei der Überwachung der mikrobiologischen Parameter und der Metalle verwendet werden ${ }^{\mathrm{a}}$

Land

\begin{tabular}{|c|c|c|c|c|}
\hline \multicolumn{5}{|l|}{ Jahr } \\
\hline Parameter & Zufallsstichprobe $^{b}$ & Stagnationsprobe ${ }^{c}$ & Spülprobe $^{d}$ & Desinfektion der Probennahmestelle \\
\hline \multicolumn{5}{|l|}{ E. coli } \\
\hline \multicolumn{5}{|l|}{ Enterococci } \\
\hline \multicolumn{5}{|l|}{ Clostridium perfringens $^{f}$} \\
\hline \multicolumn{5}{|l|}{ Coliforme Bakterien } \\
\hline \multicolumn{5}{|l|}{ Koloniezahl bei $22^{\circ} \mathrm{C}$} \\
\hline \multicolumn{5}{|l|}{ Metalle z. B. Pb, Cu, Ni } \\
\hline \multicolumn{5}{|l|}{ Freitext } \\
\hline \multicolumn{5}{|c|}{$\begin{array}{l}\text { aZutreffendes ankreuzen. 'PAls Zufallsstichprobe gilt eine Probe, die direkt vom Zapfhahn eines Verbrauchers zu einer zufälligen Tageszeit ohne vorherige Spülung aus dem } \\
\text { ersten Liter Trinkwasser genommen wird. Das Probenvolumen (bis zu } 1 \text { I) hängt vom zu untersuchenden Parameter ab. Eine Probe, die gemäß DIN EN ISO } 19458 \text { nach } \\
\text { Zweck c genommen ist, und eine Probe S-2 (vgl. Tab. A5 Anmerkung c) gelten als Zufallsstichprobe im Rahmen dieser Berichterstattung. 'Die Probennahme erfolgt nach } \\
\text { Spülung und Stagnation. Probenvolumen und Stagnationszeit bestimmen sich nach dem zu untersuchenden Parameter. 'Das Guidance document } 2011 \text { sieht hier die Mög- } \\
\text { lichkeit für Angaben vor. Aus fachlichen und rechtlichen Gründen ist die Spülprobe mit den Vorgaben der TW-RL für die zur Berichterstattung erforderlichen Probennahmen } \\
\text { allerdings nicht vereinbar. 'Desinfektion der Entnahmestelle vor der Probennahme. Eine Probe, die gemäß̈ DIN EN ISO } 19458 \text { nach Zweck b genommen worden ist, ist hier } \\
\text { zu berücksichtigen. 'Wird Clostridium perfringens auch am Wasserwerksausgang untersucht, dann informiert hierüber das BMG/UBA im Feld "Freitext." Muss nicht vom Land } \\
\text { angefüllt werden. }\end{array}$} \\
\hline
\end{tabular}

Tab. A4 Jahresübersicht über WVGa, in denen die Überwachungshäufigkeit nicht eingehalten wird ${ }^{\mathrm{b}}$. (Informationen zur Anzahl der durchgeführten Untersuchungen verglichen mit der Anzahl der erforderlichen Untersuchungen) ${ }^{b}$

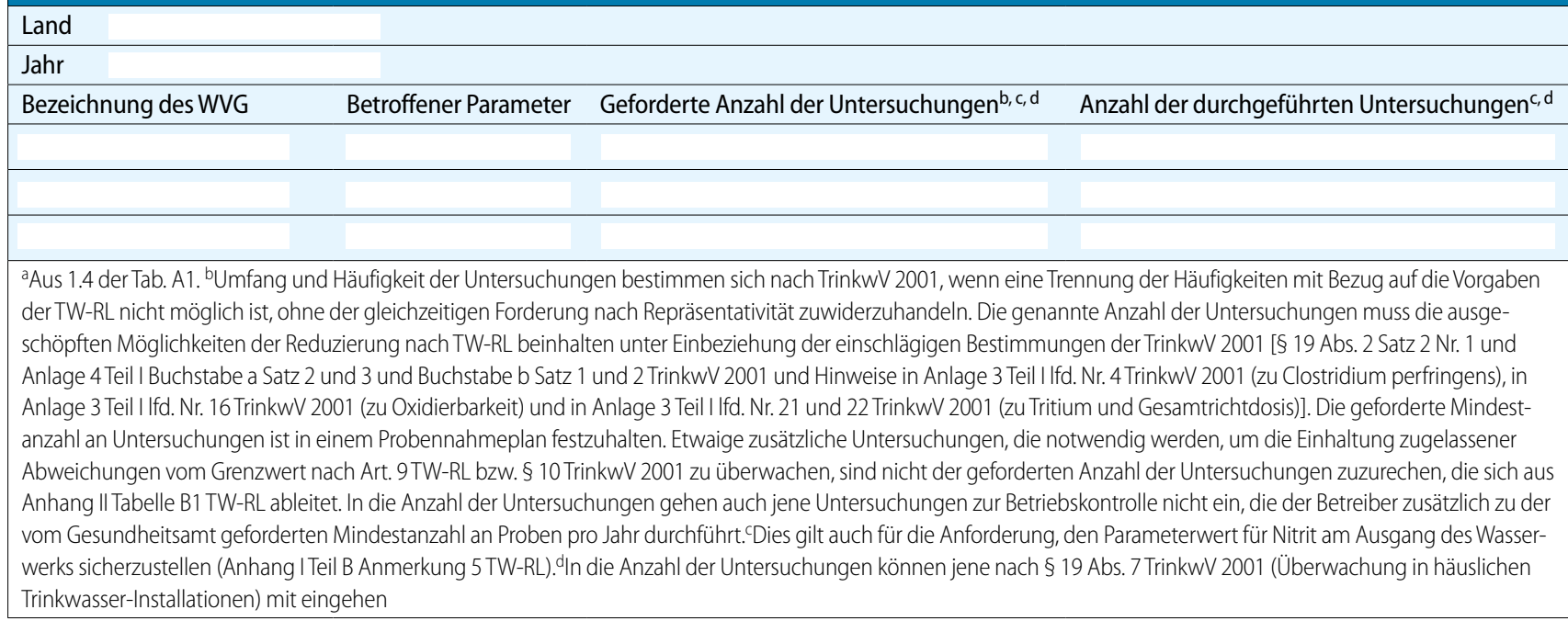


Tab. A5a Zusammenfassende Informationen über die Qualität von Wasser für den menschlichen Gebrauch in WVG, in denen mehr als $1000 \mathrm{~m}^{3}$ Wasser pro Tag verteilt oder mehr als 5000 Personen versorgt werden

Land

Jahr

Parameter

Anzahl der überwachten

WVG

$\begin{array}{ll}\text { Anzahl der } & \text { Anzahl der Un- } \\ \text { WVG mit Nicht- } & \text { tersuchungen } \\ \text { einhaltungen } & \end{array}$

Anzahl der

Untersu-

chungen mit

festgestellten

Nichteinhaltun-

gen $^{b}$

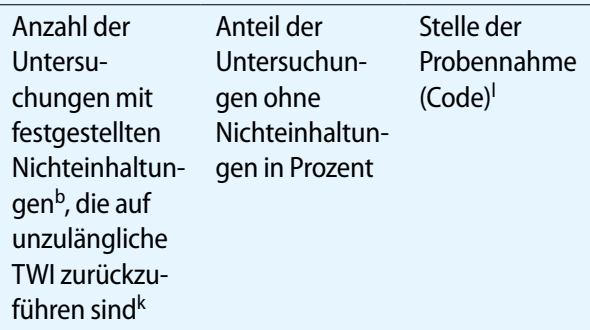

\section{Mikrobiologische}

Parameter

Escherichia coli (E. coli)

Enterokokken

Chemische Parameter

Antimon

Arsen

Benzol

Benzo-(a)-pyren

Bor

Bromat

Cadmium

Chrom

Kupfer $^{\mathrm{c}}$

Cyanid

1,2-Dichlorethan

Fluorid

Blei $^{\mathrm{c}}$

Quecksilber

Nickel $^{\mathrm{C}}$

Nitrat

Nitrit am Ausgang des

Wasserwerks

Nitrit in der Verteilung

an der Wasserentnah-

mestelle

Nitrat/Nitrit Formel

Pestizide - einzeln ${ }^{\mathrm{e}}$

Stoffname

CAS-Nummer

Pestizide insgesamt ${ }^{f}$

Polyzyklische aroma-

tische Kohlenwasser-

stoffe

Selen

Tetrachlorethan und

Trichlorethan

Trihalogenmethane -

insgesamt

Uran $^{g}$ 
Tab. A5a Zusammenfassende Informationen über die Qualität von Wasser für den menschlichen Gebrauch in WVG, in denen mehr als $1000 \mathrm{~m}^{3}$ Wasser pro Tag verteilt oder mehr als 5000 Personen versorgt werden (Fortsetzung)

Indikatorparameter

Aluminium

Ammonium

Chlorid

Clostridium

perfringens $^{\text {h }}$

Färbung

Leitfähigkeit

Wasserstoffionenkon-

zentration

Eisen

Mangan

Geruch $^{i}$

Oxidierbarkeit

Sulfat

Natrium

Geschmack $^{i}$

Koloniezahl bei $22^{\circ} \mathrm{C}$

Koloniezahl bei $36^{\circ} \mathrm{C} g$

Coliforme Bakterien

Organisch gebundener

Kohlenstoff (TOC)

Trübungi

Calcitlösekapazität ${ }^{9}$

Tritium ${ }^{j}$

Gesamtrichtdosisj

a Umfang und Häufigkeit der Untersuchungen bestimmen sich nach TrinkwV 2001. Die genannte Anzahl der Untersuchungen sollte die ausgeschöpften Möglichkeiten der Reduzierung nach TW-RL bzw. TrinkwV 2001 beinhalten (s. auch Anmerkung b in Tab. A4). In die Anzahl der Untersuchungen können jene nach § 19 Abs. 7 TrinkwV 2001 (Überwachung in Hausinstallationen) mit eingehen. 'bichteinhaltung nach TW-RL. 'Bei den Parametern Blei, Kupfer und Nickel werden die Überwachungswerte berücksichtigt, die in einer Zufallsstichprobe oder bei einer gestaffelten Probennahme in der Probe S-2 ermittelt worden sind (vgl. Empfehlung des Umweltbundesamtes zur „Beurteilung der Trinkwasserqualität hinsichtlich der Parameter Blei, Kupfer und Nickel"; Bundesgesundheitsbl Gesundheitsforsch Gesundheitsschutz 2004, 47:296-300). Die Anzahl der zu meldenden Untersuchungen leitet sich aus einem Probennahmeplan ab. ${ }^{~}$ Siehe Anlage 2 Teil II, Bemerkung in Ifd. Nr. 9 TrinkwV 2001. Die Berechnung der Formel ist die „Untersuchung". 'Die gemäß Anhang I Teil B Anmerkung 6 und 7 TW-RL gemeldete Substanz ist durch den Stoffnamen und die CAS-Nummer zu beschreiben, um eine eindeutige, vergleichbare Identifizierung der Einzelsubstanz zu erreichen. 'Pestizidkonzentrationen unterhalb der Bestimmungsgrenze sind bei der Summenberechnung nicht zu berücksichtigen. ${ }^{9}$ Hier aufgenommen, um die Anforderungen nach § 21 Abs. 2 TrinkwV 2001 zu erfüllen. Nach Anlage 3 Teil ITrinkwV 2001 hat der Parameter Koloniezahl $36^{\circ} \mathrm{C}$ keinen numerischen Parameter. ${ }^{\mathrm{h} G e m a ̈ ß ~ I f d . ~ N r . ~} 4$ der Anlage 3 Teil I TrinkwV 2001 unterrichtet die zuständige oberste Landesbehörde das Umweltbundesamt zusätzlich in Textform über das Ergebnis der Nachforschungen und Maßnahmen im Falle einer positiven Untersuchung auf Clostridium perfringens. Diese Informationen sind nach dem Format der Tab. A5d bereitzustellen. Nach Anhang I Teil C TW-RL haben die Indikatorparameter Färbung, Geruch, Geschmack, Koloniezahl $22^{\circ} \mathrm{C}$,

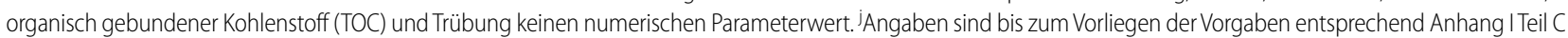
Anmerkung 10 Satz 3 und 4TW-RL freiwillig. " Angaben sind nicht verpflichtend, dienen aber ggf. der Relativierung der Ausweisung eines "WVG mit Nichteinhaltungen“. 'Hier ist ein Code aus - Tab. A7 einzutragen. Es ist möglich, dass mehr als ein Code zutrifft, wenn die Proben von verschiedenen Probennahmestellen stammen. Die Probennahmestelle muss den Vorgaben in Anhang II Tabelle B1 Satz 1 nach Untertitel der TW-RL genügen.

Tab. A5b Informationen über die produktspezifischen Parameter Acrylamid, Epichlorhydrin und Vinylchlorid

\section{Land}

Jahr

Parameter

Erläuterungen, wie die Einhaltung der Anforderungen sichergestellt ist ${ }^{\mathrm{a}}$

Acrylamid

Epichlorhydrin

Vinylchlorid

aGemäß Anhang I Teil B Anmerkung 1 TW-RL müssen die produktbezogenen Parameter Acrylamid, Epichlorhydrin und Vinylchlorid nicht im Wasser analysiert werden, dennoch muss gemäß den Produktspezifikationen des entsprechenden Polymers in Kontakt mit Trinkwasser nachgewiesen werden, dass der Parameterwert nach TW-RL eingehalten wird. Das Land erläutert, wie es diese Anforderung der TW-RL erfüllt hat, z. B. länderspezifische Regelungen für Bauprodukte in Kontakt mit Trinkwasser. Wird Trinkwasser auch auf Acrylamid, Epichlorhydrin oder Vinylchlorid chemisch analysiert, dann ist das in dieser Tabelle zu vermerken. 


\section{Tab. A5c Weitere Bemerkungen des Landes zu Tab. A5a}

Land

Jahr

Freitext

Tab. A5 da, b Information über die Ergebnisse der Nachforschungen bei Nichteinhaltung des Parameterwertes Clostridium perfringens (einschließlich Sporen) gemäß Anlage 3 Ifd. Nr. 4 TrinkwV 2001

\begin{tabular}{|c|c|c|}
\hline & & \\
\hline Jah & & \\
\hline WV & & \\
\hline & r ersten Feststellung der Nichteinhaltung & \\
\hline & e bis zur Beseitigung der Nichteinhaltung (in Tagen) & \\
\hline & $\begin{array}{l}\text { Ilte Nichteinhaltung (Anzahl/100 ml; maximal } \\
\text { rte) }\end{array}$ & \\
\hline & $\begin{array}{l}\text { e Grenzwertüberschreitungen bei coliformen } \\
\text { E. coli, Enterokokken }\end{array}$ & Ja/Nein \\
\hline & ) für die Nichteinhaltung & \\
\hline d & UR Missstände im Ressourcenschutz & \\
\hline & $\begin{array}{l}\text { UR1 Kontamination des Rohwassers mit } \\
\text { Abwässern }\end{array}$ & \\
\hline & $\begin{array}{l}\text { UR2 Kontamination des Rohwassers } \\
\text { durch Wildtiere }\end{array}$ & \\
\hline & $\begin{array}{l}\text { UR3 Zufluss kontaminierten Oberflächen- } \\
\text { wassers }\end{array}$ & \\
\hline & $\begin{array}{l}\text { UR4 Intensive Tierhaltung oder Gülleausbrin- } \\
\text { gung im Schutzgebiet }\end{array}$ & \\
\hline & UA Missstände in der Aufbereitung & \\
\hline & $\begin{array}{l}\text { UA1 Aufbereitung ohne partikelabscheidende } \\
\text { Stufe }\end{array}$ & \\
\hline & UA2 Trübungswerte über 1,0 NTU & \\
\hline & UN Undichte Rohrleitungen im Verteilungsnetz & \\
\hline $\mathrm{d}, \mathrm{e}$ & US Andere Ursachen (Beschreibung) & \\
\hline & e Maßnahmen zur Wiederherstellung der Trinkwasse & qualität \\
\hline d & MR Ressourcenschutz im Einzugsgebiet & \\
\hline & MA Optimierung der Aufbereitung & \\
\hline & MN Rohrnetzpflege & \\
\hline & $\begin{array}{l}\text { MÜ (zeitlich begrenzte) vermehrte/zusätzliche } \\
\text { Überwachung/Nachbeprobung }\end{array}$ & \\
\hline & MD Zusätzliche Desinfektion & \\
\hline d, e & MS Andere Maßnahmen (Beschreibung) & \\
\hline & & \\
\hline & $\begin{array}{l}\text { nt dazu, die Unterrichtung gemäß Anlage } 3 \text { Teil I Ifd. Nr. } 41 \\
\text { tend, wenn das vorstehende Format in der Tabelle für die } \\
\text { hrere Codes zutreffen. }{ }^{~} \text { Andere Ursache(n) oder getroffen€ }\end{array}$ & 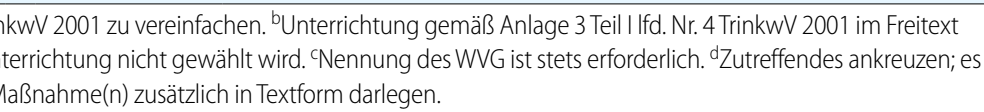 \\
\hline
\end{tabular}




\section{Bekanntmachungen - Amtliche Mitteilungen}

Tab. A6 Informationen über Nichteinhaltung der Parameterwerte bei Wasser für den menschlichen Gebrauch in WVG, in denen mehr als

$1000 \mathrm{~m}^{3}$ Wasser pro Tag verteilt oder mehr als 5000 Personen versorgt werden

Land

\begin{tabular}{|c|c|c|c|c|c|c|c|c|c|c|c|}
\hline \multicolumn{12}{|l|}{ Jahr } \\
\hline WVG & $\begin{array}{l}\text { Parame- } \\
\text { ter }\end{array}$ & $\begin{array}{l}\text { Ggf. } \\
\text { Akten- } \\
\text { zeichen } \\
\text { der zu- } \\
\text { ständigen } \\
\text { obersten } \\
\text { Landes- } \\
\text { behörde } \\
\text { für die } \\
\text { Zulassung } \\
\text { einer } \\
\text { Abwei- } \\
\text { chung }\end{array}$ & $\begin{array}{l}\text { Anzahl } \\
\text { der be- } \\
\text { troffenen } \\
\text { Bevölke- } \\
\text { rung }\end{array}$ & $\begin{array}{l}\text { Gesamt- } \\
\text { zahl der } \\
\text { Untersu- } \\
\text { chungen } \\
\text { (im Be- } \\
\text { richtsjahr) }\end{array}$ & $\begin{array}{l}\text { Anzahl } \\
\text { der Unter- } \\
\text { suchun- } \\
\text { gen mit } \\
\text { Nichtein- } \\
\text { hal- } \\
\text { tung } \\
\text { (pro Er- } \\
\text { eignis') }\end{array}$ & Max & $\begin{array}{l}\text { Med } \\
\text { total }^{d}\end{array}$ & $\begin{array}{l}\text { Med } \\
\text { noncom- } \\
\text { plient }^{\mathrm{e}}\end{array}$ & 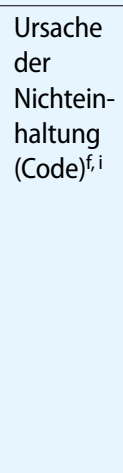 & $\begin{array}{l}\text { Abhilfe- } \\
\text { maß- } \\
\text { nahme } \\
(\text { Code) }\end{array}$ & $\begin{array}{l}\text { Zeitplan } \\
\text { (Code) }^{\mathrm{h}}\end{array}$ \\
\hline & & & & & & & & & & & \\
\hline & & & & & & & & & & & \\
\hline & & & & & & & & & & & \\
\hline $\begin{array}{l}\text { aZu ber } \\
\text { des Par } \\
\text { lassene } \\
\text { auf das } \\
\text { Untersu } \\
\text { zu, sino } \\
\text { zuziehe } \\
\text { zuordn } \\
\text { zuzuor } \\
\text { zurückf } \\
\text { Unters } \\
\text { gen. (Z }\end{array}$ & $\begin{array}{l}\text { tigen sind } \\
\text { s in Anhan } \\
\text { sichung nac } \\
\text { tsjahr oder } \\
\text { ysergebniss } \\
\text { zzeln der Ab } \\
\text { ar ist ein Co } \\
\text { er ist ein Co } \\
\text { Tritt in einer } \\
\text { dann werd } \\
\text { gen mit Nic } \\
\text { jersetzte) E }\end{array}$ & $\begin{array}{l}\text { hteinhaltung } \\
\text { eil B TW-RL. }{ }^{\circ} \\
\text { rt. } 9 \text { TW-RL fa } \\
\text { separate Erei } \\
\text { ie den Param } \\
\text { emaßnahme } \\
\text { aus Tab. A } \\
\text { aus Tab. } \\
\text { NG beim gle } \\
\text { die Ereignisse } \\
\text { nhaltung",, M } \\
\text { nisse mit glei }\end{array}$ & $\begin{array}{l}\text { vom Paran } \\
\text { u berücksich } \\
\text { (vgl. Anm } \\
\text { his (vgl. Ann } \\
\text { erwert der } \\
\text { nd dem jen } \\
\text { einzutrage } \\
10 \text { einzutrag } \\
\text { hen Parame } \\
\text { getrennter } \\
\text { kimalkonzer }\end{array}$ & $\begin{array}{l}\text { terwert jedes } \\
\text { gen sind alle } \\
\text { kung i). 'DerV } \\
\text { rkung i). 'Mec } \\
\text { (-RL nicht erfü } \\
\text { ligen Zeitrahn } \\
\text { rreffen mehre } \\
\text {. Treffen meh } \\
\text { mehr als ein } \\
\text { ailen aufgefü } \\
\text { ation" und "M }\end{array}$ & $\begin{array}{l}\text { Parameters in } \\
\text { rgebnisse, die } \\
\text { lert zeigt die } \\
\text { ianwert aller } \\
\text { llen (vgl. auch } \\
\text { hen in gesond } \\
\text { e Codes zu, sil } \\
\text { ere Codes zu, } \\
\text { Ereignis mit Ni } \\
\text { trt. Liegt meh } \\
\text { dian non-con }\end{array}$ & $\begin{array}{l}\text { Inhang I } \\
\text { die Paran } \\
\text { öchstkor } \\
\text { ntersuch } \\
\text { Anmerku } \\
\text { rten Zeil } \\
\text { d sie ein } \\
\text { ind sie e } \\
\text {-hteinha } \\
\text { als ein E } \\
\text { pliant" ( }\end{array}$ & $\begin{array}{l}\text { is C TW- } \\
\text { erte der } \\
\text { tion des } \\
\text { rgebniss } \\
\text { Hier ist e } \\
\text { uordnen } \\
\text { r Ursach } \\
\text { der Ursac } \\
\text { uf und } \\
\text { vor, danr } \\
\text { s jeweilic }\end{array}$ & $\begin{array}{l}\text { N. zugelasse } \\
\text { nicht erfüll } \\
\text { fenen Paran } \\
\text { betroffenen } \\
\text { e aus Ta } \\
\text { er Angabe } \\
\text { dem jeweili } \\
\text { d jeweiliger } \\
\text { ich die Nich } \\
\text { „_Anzahl de } \\
\text { gnis (nicht }\end{array}$ & $\begin{array}{l}\text { Abweichur } \\
\text { einschließl } \\
\text { ers im Trink } \\
\text { ameters im } \\
\text { A8 einzutra } \\
\text { Code S (Ko } \\
\text { n Zeitrahme } \\
\text { bhilfemaßn } \\
\text { nhaltunger } \\
\text { etroffenen } \\
\text { gesamte B }\end{array}$ & $\begin{array}{l}\text { n vom Par } \\
\text { jener, die } \\
\text { sser des W } \\
\text { richtsjahr. } \\
\text { n. Treffen n } \\
\text { ination an } \\
\text { ngesonde } \\
\text { ne in geso } \\
\text { fverschiec } \\
\text { ollkerung", } \\
\text { htsjahr) zu }\end{array}$ & $\begin{array}{l}\text { terwert je- } \\
\text { r eine zuge- } \\
\text { an, bezogen } \\
\text { dianwert der } \\
\text { rere Codes } \\
\text { sachen) vor- } \\
\text { Z Zeilen zu- } \\
\text { erten Zeilen } \\
\text { e Ursachen } \\
\text { zahl der } \\
\text { ücksichti- }\end{array}$ \\
\hline
\end{tabular}

Tab. A7 Codes für die Stelle der Probennahme

\begin{tabular}{ll} 
Code & Stelle der Probennahme \\
\hline W & Wasserwerk(sausgang) \\
\hline $\mathrm{N}$ & Öffentliches Verteilungsnetz \\
\hline $\mathrm{L}$ & Stelle der Übergabe aus dem Verteilungsnetz des Wasserversorgers in die Trinkwasser-Installation \\
\hline $\mathrm{T}$ & Häusliche Trinkwasserinstallation \\
\hline
\end{tabular}

Tab. A8 Codes für die Ursachen einer Nichteinhaltung

\begin{tabular}{ll}
\hline Code & Ursache \\
\hline C & Auf Einzugsgebiet zurückzuführen \\
\hline$T$ & Auf Wasserwerk zurückzuführen \\
\hline P & Auf öffentliches Verteilungsnetz zurückzuführen \\
\hline D & Auf häusliche Trinkwasser-Installation zurückzuführen \\
\hline O & Andere Ursache \\
\hline S & Kombination an Ursachen \\
\hline U & Unbekannte Ursache \\
\hline
\end{tabular}


Tab. A9 Codes für Abhilfemaßnahmen bei Nichteinhaltung

\begin{tabular}{|c|c|}
\hline Code & Beschreibung der Abhilfemaßnahme \\
\hline \multicolumn{2}{|c|}{ Cfür Einzugsgebiet } \\
\hline C1 & Maßnahme(n) zur Behebung bzw. Minderung der Ursache \\
\hline $\mathrm{C} 2$ & Maßnahme(n) zum Ersatz der Wasserressource \\
\hline \multicolumn{2}{|c|}{ Tfür Aufbereitung } \\
\hline $\mathrm{T} 1$ & Einführung, Aufrüstung oder Ertüchtigung der Aufbereitung \\
\hline \multicolumn{2}{|c|}{ P für öffentliches Verteilungsnetz } \\
\hline P1 & Austausch, Abtrennung oder Reparatur defekter Teile \\
\hline P2 & Mechanische/chemische Reinigung und/oder Desinfektion verunreinigter Teile \\
\hline \multicolumn{2}{|c|}{ D für Trinkwasser-Installation ${ }^{\mathrm{a}}$} \\
\hline D1 & Austausch, Abtrennung oder Reparatur defekter Teile \\
\hline D2 & Mechanische/chemische Reinigung und/oder Desinfektion verunreinigter Teile \\
\hline \multicolumn{2}{|c|}{ SSicherungsmaßnahmen, um Zutritt durch Unbefugte zu verhindern } \\
\hline S1 & Sicherungsmaßnahmen, um Zutritt durch Unbefugte zu verhindern \\
\hline \multicolumn{2}{|c|}{ OSonstiges } \\
\hline 01 & Sonstiges \\
\hline \multicolumn{2}{|c|}{ E Notfallmaßnahmen im Sinne der Verbrauchergesundheit und -sicherheit } \\
\hline E1 & $\begin{array}{l}\text { Benachrichtigung der Verbraucher und Anweisungen (z. B. Entnahmeverbot, Abkochgebot, vorüberge- } \\
\text { hende Gebrauchseinschränkung) }\end{array}$ \\
\hline E2 & $\begin{array}{l}\text { Vorübergehende Bereitstellung einer alternativen Trinkwasserversorgung (z. B. Wasser in Flaschen, Be- } \\
\text { hältern, Tankwagen) }\end{array}$ \\
\hline
\end{tabular}

\section{Tab. A10 Codes für den Zeitrahmen der Abhilfemaßnahmen}

\begin{tabular}{|ll}
\hline Code & Zeitrahmen \\
\hline I & Sehr kurzfristig, d. h. nicht mehr als 1 Tag \\
\hline S & Kurzfristig, d. h. nicht mehr als 30 Tage \\
\hline M & Mittelfristig, d. h. mehr als 30 Tage, aber nicht mehr als 1 Jahr \\
\hline L & Langfristig, d. h. mehr als 1 Jahr \\
\hline
\end{tabular}

Tab. A11 Kommunikation der im Land verfügbaren Informationen über die Qualität von Wasser für den menschlichen Gebrauch

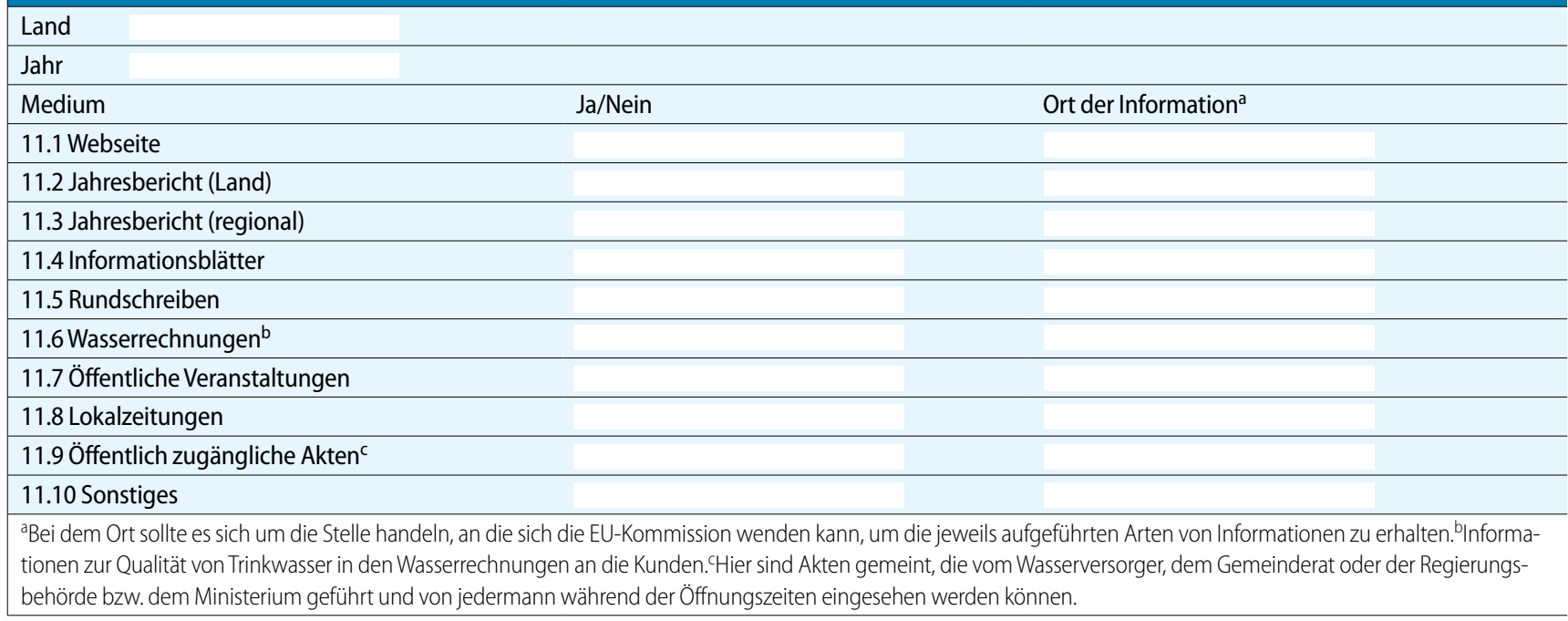




\section{Bekanntmachungen - Amtliche Mitteilungen}

Tab. A12 Art der im Land öffentlich zugänglichen Informationen

\section{Land}

Jahr

Art der verfügbaren Informationen

Ja/Nein

Ort der Information $^{\text {a }}$

12.1 Landesweite Zusammenfassung über die Einhaltung der TW-RL

12.2 Regionale Zusammenfassung über die Einhaltung der TW-RL

12.3 Zusammenfassung der Einhaltung der TW-RL bezogen auf das WVG

12.4 Einzelne Überwachungsergebnisse für das WVG

12.5 Überwachungsergebnisse für kleine WVG (in denen im Durchschnitt 10

bis $1000 \mathrm{~m}^{3} /$ Tag verteilt und 50 bis 5000 Personen versorgt werden)

12.6 Überwachungsergebnisse für sehr kleine WVG (in denen im Durchschnitt weniger als $10 \mathrm{~m}^{3} /$ Tag verteilt oder weniger als 50 Personen versorgt werden)

12.7 Informationen über Einhaltung strengerer nationaler Standards

12.8 Informationen über Einhaltung zusätzlicher nationaler Standards

12.9 Informationen auf der Ebene eines WVG über Nichteinhaltungen

12.10 Informationen über die Trinkwasserressource $(n)$ im betreffenden WVG

12.11 Sonstiges

aBei dem Ort sollte es sich um die Stelle handeln, an die sich die EU-Kommission wenden kann, um die jeweils aufgeführten Arten von Informationen zu erhalten. 


\section{Berichterstattung gemäß Trinkwasserverordnung ${ }^{6}$}

\subsection{Rechtliche Grundlage und allgemeine Anforderungen}

Gemäß $\$ 21$ Absatz 2 TrinkwV 2001 berichten die zuständigen obersten Landesbehörden nach den Formatvorgaben der EU-Kommission über die Trinkwasserbeschaffenheit in Wasserversorgungsgebieten (WVG), in denen mindestens $10 \mathrm{~m}^{3}$ Wasser pro Tag geliefert oder mindestens 50 Personen versorgt werden. Dies verlangt, die Mindestanforderungen in der Trinkwasserberichterstattung auf kleinere als in der TW-RL festgelegte WVG anzuwenden. Bund und Länder haben sich darauf verständigt, für die WVG mit dem TrinkwV-spezifischen Größenbereich aus praktischen Erwägungen ein separates Formblatt zu verwenden.

Das Format übernimmt im Wesentlichen die Vorgaben der Berichterstattung nach TW-RL. Es legt dafür die Grenzwerte und Anforderungen der TrinkwV 2001 zugrunde und berücksichtigt die aus der TrinkwV 2001 resultierenden Besonderheiten. Die unter 2.1 erwähnten Grundsätze 2-1 bis 2-11 gelten entsprechend mit der Einschränkung, dass

- die Landesbehörden an das UBA jährlich über die Beschaffenheit von Trinkwasser aus WVG berichten, in denen im Durchschnitt mindestens $10 \mathrm{~m}^{3}$ Wasser pro Tag verteilt oder mindestens 50 Personen versorgt werden sowie höchstens $1000 \mathrm{~m}^{3}$ Wasser pro Tag verteilt und höchstens 5000 Personen versorgt werden,

- Datenerfassung und Datenbereitstellung zwar den Vorgaben der EUKommission für europäische Berichtspflichten genügen sollen, bislang aber die routinemäßige Berichterstattung an die EU-Kommission nicht festgeschrieben ist, und

- für die Mitteilungen und Informationen über zugelassene Abweichungen die Festlegungen in $\$ 10$ TrinkwV 2001 gelten, Angaben aus diesen Mitteilungen und Informationen auch Bestandteil der Berich-

${ }^{6}$ Hier werden die WVG berücksichtigt, über die nicht bereits nach TW-RL zu berichten ist. te über die Trinkwasserbeschaffenheit in WVG mit TrinkwV-spezifischem Größenbereich sind und den Mitteilungen und Informationen nach $\$ 10$ TrinkwV 2001 die in Anlage 2 TrinkwV 2001 genannten Grenzwerte zugrunde liegen.

Die obersten Landesbehörden berichten dem UBA ab dem Berichtsjahr 2014 nach dem hier vorgegebenen Format in der in $\$ 21$ Absatz 2 TrinkwV 2001 genannten Frist und stellen den Bericht als XML-Datei über die für Trinkwasser spezifizierte XML-Schnittstelle in die zentrale Datenhaltung des „WasserBLIcK“ bei der BfG ein.

\subsection{Informationen, die dem Umweltbundesamt jährlich vorzulegen sind:}

\subsubsection{Informationen über die allgemeinen Rahmenbedingungen zur Trinkwasserversorgung im jeweiligen Land}

Das erforderliche Format ist durch - Tab. B1 bestimmt. ${ }^{7}$

\subsubsection{Informationen über Probennahmeverfahren, die bei der Überwachung der mikrobiologischen Parameter und der Metalle eingesetzt werden}

Diesbezügliche Angaben sind in - Tab. B3b nur einzutragen, wenn sie im Berichtsjahr von der Berichterstattung nach TW-RL nicht ausreichend berücksichtigt werden.

\subsubsection{Kurzinformationen aus der Überwachung in Wasserversorgungsgebieten}

Diese Informationen sind nach dem Format der $\bullet$ Tab. B4 bereitzustellen.

\footnotetext{
7 Die Tabellennummerierung folgt dem Guidance document (s. Fußnote 4). Der hinzugefügte Großbuchstabe dient der besseren Zuordnung der im nationalen Format verwendeten Tabellen. Die Informationen, die das Guidance document in Tab. 2 und 3a einfordert, erteilen BMG und UBA.
}

\subsubsection{Informationen zur Trinkwasserqualität in Wasserversorgungsgebieten auf landesweiter Ebene}

Diese Informationen sind in dem Format gemäß Tab. B5a wiederzugeben (Raum für weitere, auch fakultative Angaben zu Tab. B5a bietet $\bullet$ Tab. B5c).

\subsubsection{Informationen nach dem Format der 0 Tab. B5b über die produktspezifischen Parameter Acrylamid, Epichlorhydrin und Vinylchlorid}

\subsubsection{Information über die Ergebnisse der Nachforschungen bei Nichteinhaltung des Parameterwertes Clostridium perfringens \\ Dafür ist $\bullet$ Tab. B5d heranzuziehen.}

\subsubsection{Informationen zu Nichteinhaltungen von Parameterwerten in den unter 1.4.1 in Tab. B1 bezeichneten und betroffenen Wasserversorgungsgebieten}

Diese Informationen müssen den Vorgaben gemäß • Tab. B6 entsprechen. Hier sind auch jene zugelassenen Abweichungen im Berichtsjahr zu berücksichtigen, über die das Umweltbundesamt bereits nach $\$ 10$ TrinkwV 2001 von den zuständigen obersten Landesbehörden unterrichtet worden ist.

\subsection{Informationen über die \\ Qualität von Trinkwasser an die Öffentlichkeit (vgl. BMG-Mitteilung 2008, Kapitel 4)}

Diese Informationen sind nur dann nach dem Format der $\bullet$ Tab. B11 und B12 zu liefern, wenn diesbezügliche Angaben für das Berichtsjahr nicht oder nicht vollständig nach dem Format der Tab. A11 und A12 bereitgestellt werden. 


\section{Bekanntmachungen - Amtliche Mitteilungen}

Tab. B1 Allgemeine Informationen über die Rahmenbedingungen der Trinkwasserversorgung in dem Land. (Mit Ausnahme von Frage 1.2 werden nur Informationen über WVG gemeldet, in denen mindestens $10 \mathrm{~m}^{3}$ Trinkwasser pro Tag verteilt oder mindestens 50 Personen versorgt werden sowie höchstens $1000 \mathrm{~m}^{3}$ Trinkwasser pro Tag verteilt und höchstens 5000 Personen versorgt werden)

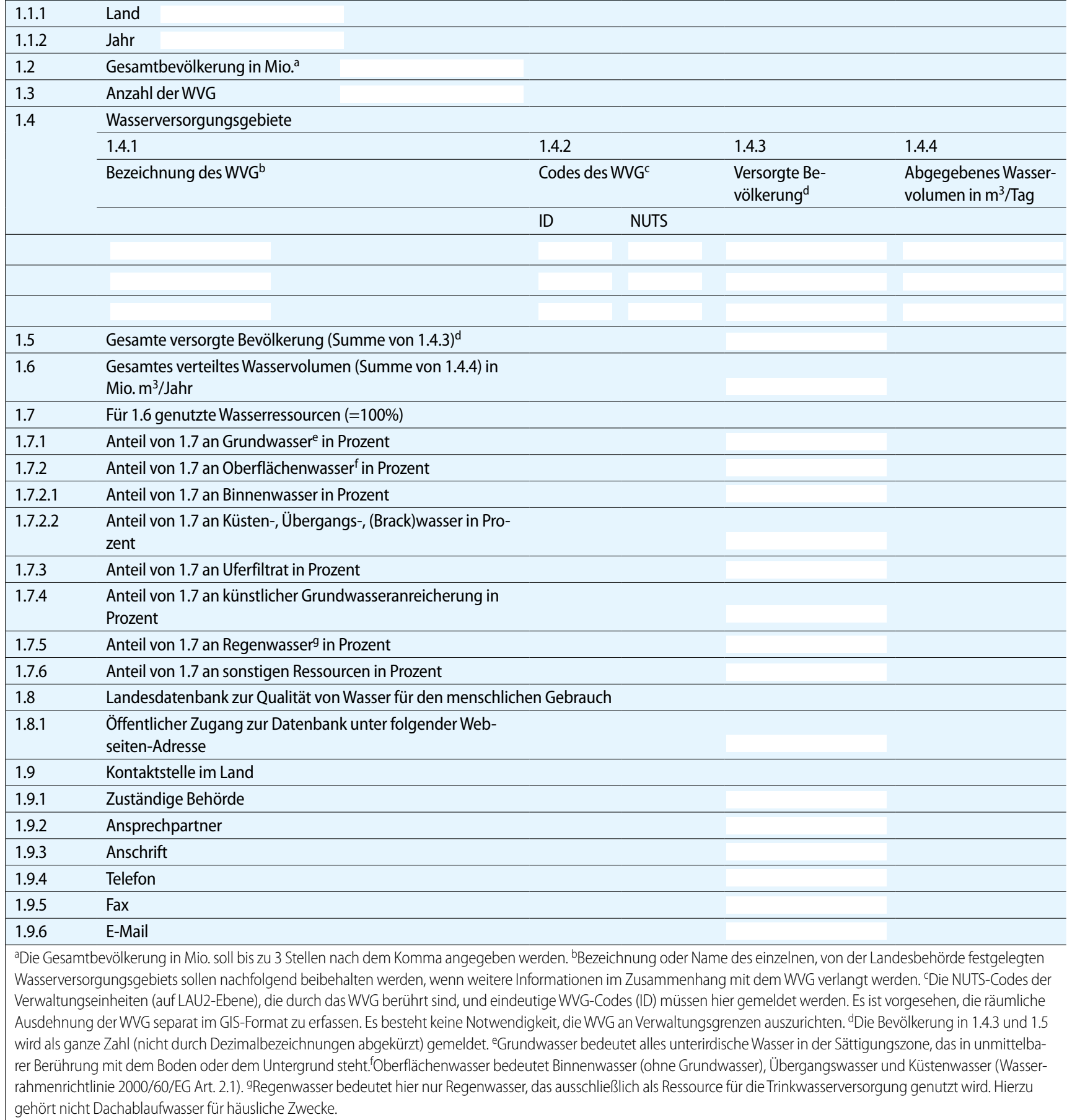


Tab. B3b Probennahmeverfahren, die bei der Überwachung der mikrobiologischen Parameter und der Metalle verwendet werden ${ }^{\mathrm{a}}$. (Angaben hier fakultativ, wenn sie im Berichtsjahr von der Berichterstattung nach TW-RL ${ }^{\mathrm{b}}$ ausreichend berücksichtigt sind)

\begin{tabular}{|c|c|c|c|c|}
\hline \multicolumn{5}{|l|}{ Jahr } \\
\hline Parameter & Zufallsstichprobe $^{c}$ & Stagnationsprobe ${ }^{d}$ & Spülprobe ${ }^{\mathrm{e}}$ & Desinfektion der Probennahmestelle ${ }^{f}$ \\
\hline \multicolumn{5}{|l|}{ E. coli } \\
\hline \multicolumn{5}{|l|}{ Enterococci } \\
\hline \multicolumn{5}{|l|}{ Clostridium perfringens ${ }^{g}$} \\
\hline \multicolumn{5}{|l|}{ Coliforme Bakterien } \\
\hline \multicolumn{5}{|l|}{ Koloniezahl bei $22^{\circ} \mathrm{C}$} \\
\hline \multicolumn{5}{|l|}{ Metalle, z. B. Pb, Cu, Ni } \\
\hline \multicolumn{5}{|l|}{ Freitext } \\
\hline \multicolumn{5}{|c|}{ 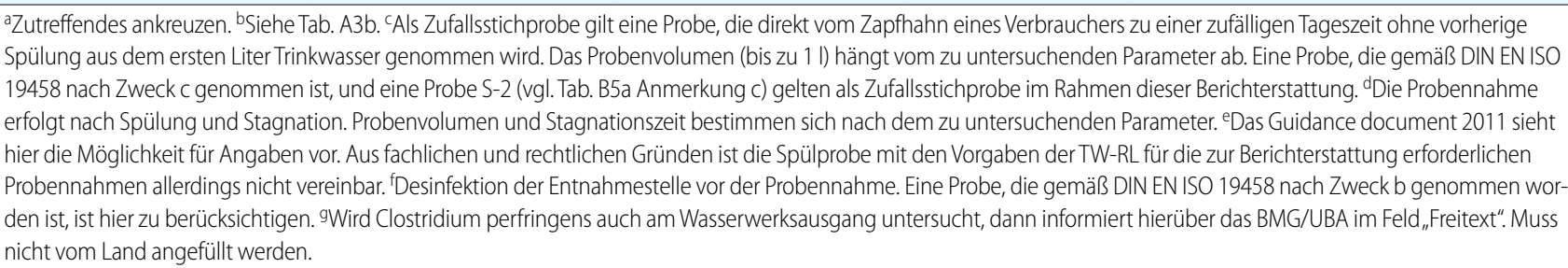 } \\
\hline
\end{tabular}

Tab. B4 Jahresübersicht über WVG ${ }^{a}$, in denen die Überwachungshäufigkeit nicht eingehalten wird ${ }^{b}$. (Informationen zur Anzahl der durchgeführten Untersuchungen verglichen mit der Anzahl der erforderlichen Untersuchungen) ${ }^{\mathrm{b}}$

Land

Jahr

Bezeichnung des WVG Betroffener Parameter Geforderte Anzahl der Untersuchungen ${ }^{b, c, d} \quad$ Anzahl der durchgeführten Untersuchungen ${ }^{c, d}$

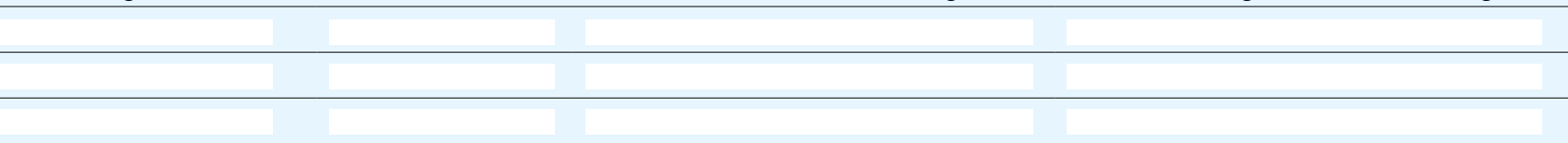

${ }^{a}$ Aus 1.4 der Tab. 1. 'bumfang und Häufigkeit der Untersuchungen bestimmen sich nach TrinkwV 2001. Die genannte Anzahl der Untersuchungen muss die ausgeschöpften Möglichkeiten der Reduzierung nach TrinkwV 2001 beinhalten [vgl. § 19 Abs. 2 Satz 2 Nr. 1 und Anlage 4 Teil I Buchstabe a Satz 2 und 3 und Buchstabe b Satz 1 und 2 TrinkwV 2001 und Hinweise in Anlage 3 Teil I lfd. Nr. 4 TrinkwV 2001 (zu Clostridium perfringens), in Anlage 3 Teil I lfd. Nr. 16 TrinkwV 2001 (zu Oxidierbarkeit) und in Anlage 3 Teil I ffd. Nr. 21 und 22 TrinkwV 2001 (zu Tritium und Gesamtrichtdosis)]. Die geforderte Mindestanzahl an Untersuchungen ist in einem Probennahmeplan festzuhalten. Etwaige zusätzliche Untersuchungen, die notwendig werden, um die Einhaltung zugelassener Abweichungen vom Grenzwert nach § 10 TrinkwV 2001 zu überwachen, sind nicht der geforderten Anzahl der Untersuchungen zuzurechnen. In die Anzahl der Untersuchungen gehen auch jene Untersuchungen zur Betriebskontrolle nicht ein, die der Betreiber zusätzlich zu der vom Gesundheitsamt geforderten Mindestanzahl an Proben pro Jahr durchführt. 'Dies gilt auch für die Anforderung, den Parameterwert für Nitrit am Ausgang des Wasserwerks sicherzustellen (Anlage 2 Teil II Bemerkung in Ifd. Nr. 9 TrinkwV 2001). In die Anzahl der Untersuchungen können jene nach § 19 Abs. 7 TrinkwV 2001 (Überwachung in häuslichen Trinkwasser-Installationen) mit eingehen. 


\section{Bekanntmachungen - Amtliche Mitteilungen}

Tab. B5a Zusammenfassende Informationen über die Qualität von Trinkwasser in WVG, in denen mindestens $10 \mathrm{~m}^{3}$ Trinkwasser pro Tag ver-

teilt oder mindestens 50 Personen versorgt werden sowie höchstens $1000 \mathrm{~m}^{3}$ Trinkwasser pro Tag verteilt und höchstens 5000 Personen versorgt werden

\begin{tabular}{|c|c|c|c|c|c|c|c|}
\hline Jahr & & & & & & & \\
\hline Parameter & $\begin{array}{l}\text { Anzahl der über- } \\
\text { wachten WVG }\end{array}$ & $\begin{array}{l}\text { Anzahl der } \\
\text { WVG mit } \\
\text { Nichteinhal- } \\
\text { tungen }\end{array}$ & $\begin{array}{l}\text { Anzahl der Un- } \\
\text { tersuchungen }{ }^{a}\end{array}$ & $\begin{array}{l}\text { Anzahl der } \\
\text { Untersu- } \\
\text { chungen mit } \\
\text { festgestellten } \\
\text { Nichteinhal- } \\
\text { tungen }^{b}\end{array}$ & $\begin{array}{l}\text { Anzahl der } \\
\text { Untersu- } \\
\text { chungen mit } \\
\text { festgestellten } \\
\text { Nichteinhal- } \\
\text { tungen }{ }^{\text {b }} \text {, die } \\
\text { auf unzuläng- } \\
\text { liche TWl zu- } \\
\text { rückzuführen } \\
\text { sindj }\end{array}$ & $\begin{array}{l}\text { Anteil der } \\
\text { Untersuchun- } \\
\text { gen ohne Nicht- } \\
\text { einhaltungen in } \\
\text { Prozent }\end{array}$ & $\begin{array}{l}\text { Stelle der } \\
\text { Probennah- } \\
\text { me (Code) }\end{array}$ \\
\hline \multicolumn{8}{|l|}{$\begin{array}{l}\text { Mikrobiologische Para- } \\
\text { meter }\end{array}$} \\
\hline \multicolumn{8}{|l|}{ Escherichia coli (E. coli) } \\
\hline \multicolumn{8}{|l|}{ Enterokokken } \\
\hline \multicolumn{8}{|l|}{ Chemische Parameter } \\
\hline \multicolumn{8}{|l|}{ Antimon } \\
\hline \multicolumn{8}{|l|}{ Arsen } \\
\hline \multicolumn{8}{|l|}{ Benzol } \\
\hline \multicolumn{8}{|l|}{ Benzo-(a)-pyren } \\
\hline \multicolumn{8}{|l|}{ Bor } \\
\hline \multicolumn{8}{|l|}{ Bromat } \\
\hline \multicolumn{8}{|l|}{ Cadmium } \\
\hline \multicolumn{8}{|l|}{ Chrom } \\
\hline \multicolumn{8}{|l|}{ Kupfer ${ }^{c}$} \\
\hline \multicolumn{8}{|l|}{ Cyanid } \\
\hline \multicolumn{8}{|l|}{ 1,2-Dichlorethan } \\
\hline \multicolumn{8}{|l|}{ Fluorid } \\
\hline \multicolumn{8}{|l|}{ Blei $^{c}$} \\
\hline \multicolumn{8}{|l|}{ Quecksilber } \\
\hline \multicolumn{8}{|l|}{ Nickel $^{c}$} \\
\hline \multicolumn{8}{|l|}{ Nitrat } \\
\hline \multicolumn{8}{|l|}{$\begin{array}{l}\text { Nitrit am Ausgang des } \\
\text { Wasserwerks }\end{array}$} \\
\hline \multicolumn{8}{|l|}{$\begin{array}{l}\text { Nitrit in der Verteilung } \\
\text { an der Wasserentnahme- } \\
\text { stelle }\end{array}$} \\
\hline \multicolumn{8}{|l|}{ Nitrat/Nitrit Formel ${ }^{d}$} \\
\hline \multicolumn{8}{|l|}{$\begin{array}{l}\text { Pestizide - einzeln }{ }^{\mathrm{e}} \\
\text { Stoffname }\end{array}$} \\
\hline \multicolumn{8}{|l|}{ CAS-Nummer } \\
\hline \multicolumn{8}{|l|}{ Pestizide insgesamt ${ }^{f}$} \\
\hline \multicolumn{8}{|l|}{$\begin{array}{l}\text { Polyzyklische aromati- } \\
\text { sche Kohlenwasserstoffe }\end{array}$} \\
\hline \multicolumn{8}{|l|}{ Selen } \\
\hline \multicolumn{8}{|l|}{$\begin{array}{l}\text { Tetrachlorethan und } \\
\text { Trichlorethan }\end{array}$} \\
\hline $\begin{array}{l}\text { Trihalogenmethane - } \\
\text { insgesamt }\end{array}$ & & & & & & & \\
\hline Uran & & & & & & & \\
\hline
\end{tabular}


Tab. B5a Zusammenfassende Informationen über die Qualität von Trinkwasser in WVG, in denen mindestens $10 \mathrm{~m}^{3}$ Trinkwasser pro Tag verteilt oder mindestens 50 Personen versorgt werden sowie höchstens $1000 \mathrm{~m}^{3}$ Trinkwasser pro Tag verteilt und höchstens 5000 Personen versorgt werden (Fortsetzung)

\section{Indikatorparameter}

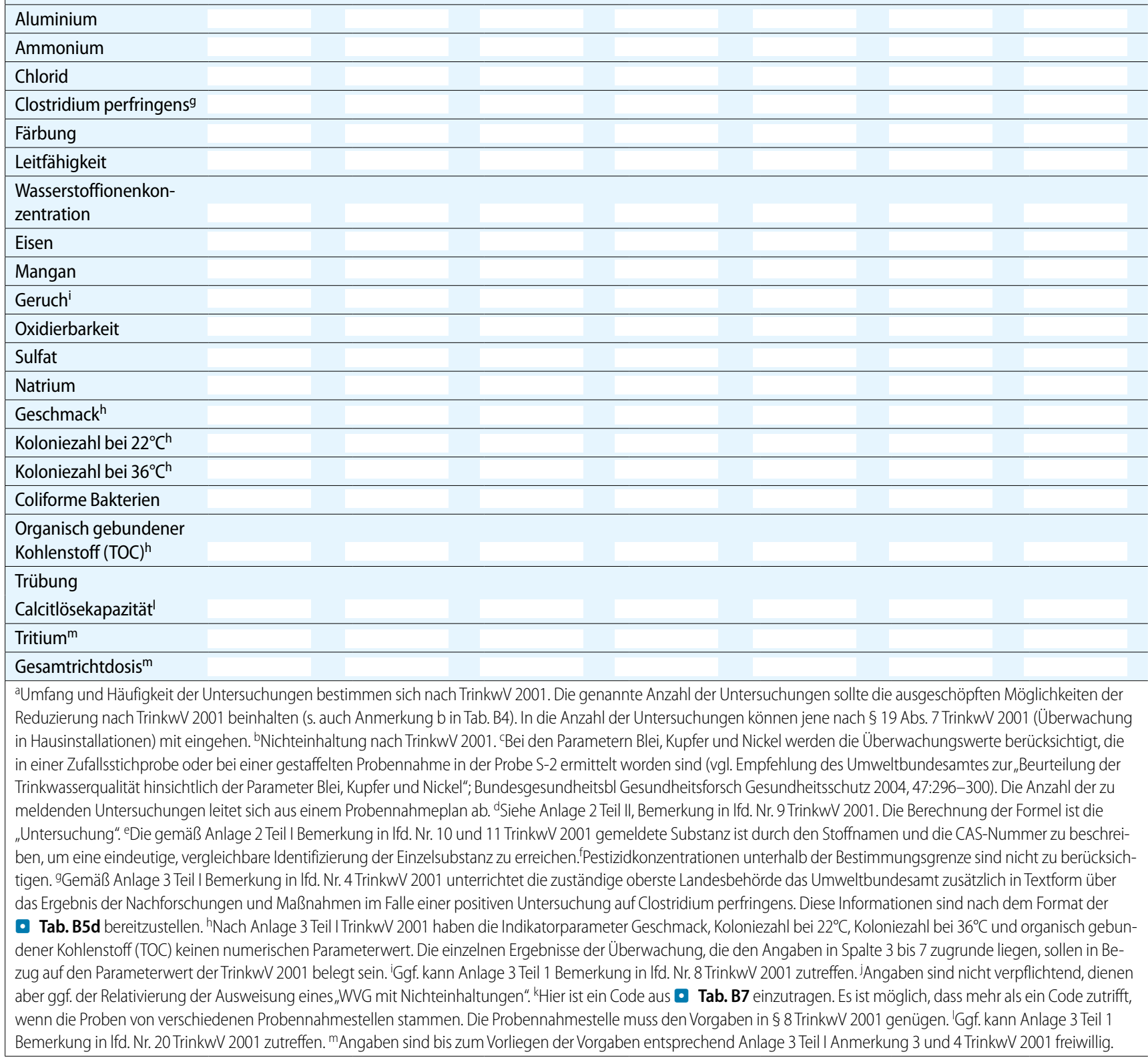

Tab. B5b Informationen über die produktspezifischen Parameter Acrylamid, Epichlorhydrin und Vinylchlorid

Land

Jahr

Parameter Erläuterungen, wie die Einhaltung der Anforderungen sichergestellt ist ${ }^{\mathrm{a}}$

Acrylamid

Epichlorhydrin

Vinylchlorid

áGemäß Anlage 3 Teil I Bemerkung in Ifd. Nr. 1 und Teil II Bemerkungen in Ifd. Nr. 6 und 12 TrinkwV 2001 müssen die produktbezogenen Parameter Acrylamid, Epichlorhydrin und Vinylchlorid nicht im Wasser analysiert werden, dennoch muss gemäß den Produktspezifikationen des entsprechenden Polymers in Kontakt mit Trinkwasser nachgewiesen werden, dass der Parameterwert nach TrinkwV 2001 eingehalten wird. Das Land erläutert, wie es diese Anforderung der TrinkwV 2001 erfüllt hat, z. B. länderspezifische Regelungen für Bauprodukte in Kontakt mit Trinkwasser. Wird Trinkwasser auch auf Acrylamid, Epichlorhydrin oder Vinylchlorid chemisch analysiert, dann ist das in dieser Tabelle zu vermerken. 


\section{Bekanntmachungen - Amtliche Mitteilungen}

\section{Tab. B5c Weitere Bemerkungen des Landes zu Tab. B5a}

Land

Jahr

Freitext

Tab. B5 $\mathbf{d}^{\mathbf{a}, \mathbf{b}}$ Information über die Ergebnisse der Nachforschungen bei Nichteinhaltung des Parameterwertes Clostridium perfringens (ein-

schließlich Sporen) gemäß Anlage 3 Ifd. Nr. 4 TrinkwV 2001

Land

Jahr

WVGc

Datum der ersten Feststellung der Nichteinhaltung

Zeitspanne bis zur Beseitigung der Nichteinhaltung

(in Tagen)

Festgestellte Nichteinhaltung (Anzahl/100 ml; maximal 3 Messwerte)

Zusätzliche Grenzwertüberschreitungen bei coliformen Ja/Nein

Bakterien, E. coli, Enterokokken

Ursache(n) für die Nichteinhaltung

d UR Missstände im Ressourcenschutz

UR1 Kontamination des Rohwassers mit

Abwässern

UR2 Kontamination des Rohwassers durch

Wildtiere

UR3 Zufluss kontaminierten Oberflächen-

wassers

UR4 Intensive Tierhaltung oder Gülleaus-

bringung im Schutzgebiet

UA Missstände in der Aufbereitung

UA1 Aufbereitung ohne partikelabscheidende Stufe

UA2 Trübungswerte über 1,0 NTU

UN Undichte Rohrleitungen im

Verteilungsnetz

$\mathrm{d}, \mathrm{e} \quad$ US Andere Ursachen (Beschreibung)

Getroffene Maßnahmen zur Wiederherstellung der Trinkwasserqualität

d MR Ressourcenschutz im Einzugsgebiet

MA Optimierung der Aufbereitung

MN Rohrnetzpflege

MÜ (zeitlich begrenzte) vermehrte/zusätzliche

Überwachung/Nachbeprobung

MD Zusätzliche Desinfektion

d, e MS Andere Maßnahmen (Beschreibung)

Freitext ${ }^{b}$

aTabelle dient dazu, die Unterrichtung gemäß Anlage 3 Teil I lfd. Nr. 4 TrinkwV 2001 zu vereinfachen. bUnterrichtung gemäß Anlage 3 Teil I lfd. Nr. 4 TrinkwV 2001 im Freitext

ist verpflichtend, wenn das vorstehende Format in der Tabelle für die Unterrichtung nicht gewählt wird. 'Nennung des WVG ist stets erforderlich. 'ंZutreffendes ankreuzen; es

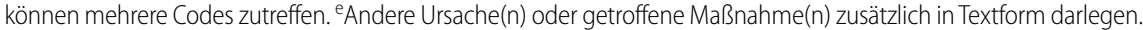


Tab. B6 Informationen über Nichteinhaltung der Parameterwerte bei Trinkwasser in WVG, in denen mindestens $10 \mathrm{~m}^{3}$ Trinkwasser pro Tag verteilt oder mindestens 50 Personen versorgt werden sowie höchstens $1000 \mathrm{~m}^{3}$ Trinkwasser pro Tag verteilt und höchstens 5000 Personen versorgt werden

\begin{tabular}{|c|c|c|c|c|c|c|c|c|c|c|c|}
\hline \multicolumn{12}{|l|}{ Land } \\
\hline \multicolumn{12}{|l|}{ Jahr } \\
\hline WVG & $\begin{array}{l}\text { Parame- } \\
\text { ter }\end{array}$ & $\begin{array}{l}\text { Ggf. } \\
\text { Akten- } \\
\text { zeichen } \\
\text { der zu- } \\
\text { ständigen } \\
\text { obersten } \\
\text { Landes- } \\
\text { behörde } \\
\text { für die } \\
\text { Zulassung } \\
\text { einer } \\
\text { Abwei- } \\
\text { chung }^{\text {a }}\end{array}$ & $\begin{array}{l}\text { Anzahl } \\
\text { der be- } \\
\text { troffenen } \\
\text { Bevölke- } \\
\text { rung }\end{array}$ & $\begin{array}{l}\text { Gesamt- } \\
\text { zahl der } \\
\text { Untersu- } \\
\text { chungen } \\
\text { (im Be- } \\
\text { richtsjahr) }\end{array}$ & $\begin{array}{l}\text { Anzahl } \\
\text { der Unter- } \\
\text { suchun- } \\
\text { gen mit } \\
\text { Nichtein- } \\
\text { haltunga, } \\
\text { b (pro } \\
\text { Ereignis') }\end{array}$ & Max & $\begin{array}{l}\text { Med } \\
\text { total }^{d}\end{array}$ & $\begin{array}{l}\text { Med } \\
\text { noncom- } \\
\text { plient }^{\mathrm{e}}\end{array}$ & $\begin{array}{l}\text { Ursache } \\
\text { der } \\
\text { Nichtein- } \\
\text { haltung } \\
\text { (Code) })^{f, i}\end{array}$ & $\begin{array}{l}\text { Abhilfe- } \\
\text { maß- } \\
\text { nahme } \\
\text { (Code) }^{g}\end{array}$ & 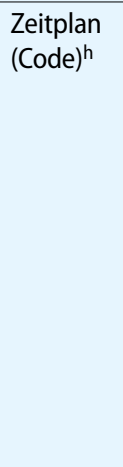 \\
\hline & & & & & & & & & & & \\
\hline & & & & & & & & & & & \\
\hline & & & & & & & & & & & \\
\hline \multicolumn{12}{|c|}{ 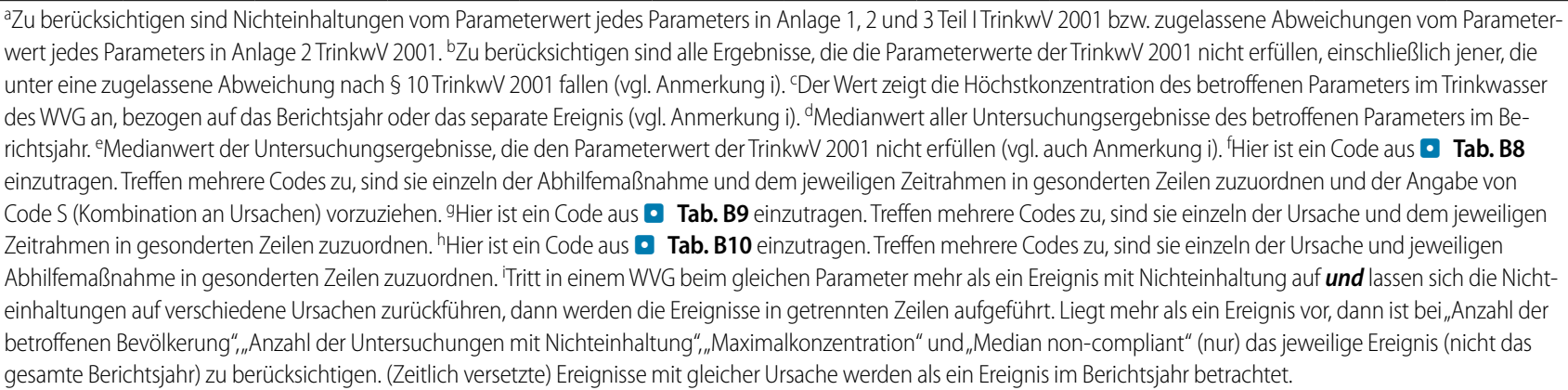 } \\
\hline
\end{tabular}

\section{Tab. B7 Codes für die Stelle der Probennahme}

\begin{tabular}{|ll}
\hline Code & Stelle der Probennahme \\
\hline W & Wasserwerk(sausgang) \\
\hline $\mathrm{N}$ & Öffentliches Verteilungsnetz \\
\hline $\mathrm{L}$ & Stelle der Übergabe aus dem Verteilungsnetz des Wasserversorgers in die Trinkwasser-Installation \\
\hline $\mathrm{T}$ & Häusliche Trinkwasser-Installation \\
\hline
\end{tabular}

\section{Tab. B8 Codes für die Ursachen einer Nichteinhaltung}

\begin{tabular}{ll}
\hline Code & Ursache \\
\hline C & Auf Einzugsgebiet zurückzuführen \\
\hline$T$ & AufWasserwerk zurückzuführen \\
\hline$P$ & Auf öffentliches Verteilungsnetz zurückzuführen \\
\hline$D$ & Auf häusliche Trinkwasser-Installation zurückzuführen \\
\hline O & Andere Ursache \\
\hline S & Kombination an Ursachen \\
\hline$U$ & Unbekannte Ursache \\
\hline
\end{tabular}




\section{Bekanntmachungen - Amtliche Mitteilungen}

\section{Tab. B9 Codes für Abhilfemaßnahmen bei Nichteinhaltung}

Code Beschreibung der Abhilfemaßnahme

C für Einzugsgebiet

C1 Maßnahme(n) zur Behebung bzw. Minderung der Ursache

C2 Maßnahme(n) zum Ersatz der Wasserressource

Tfür Aufbereitung

T1 Einführung, Aufrüstung oder Ertüchtigung der Aufbereitung

P für öffentliches Verteilungsnetz

P1 Austausch, Abtrennung oder Reparatur defekter Teile

P2 Mechanische/chemische Reinigung und/oder Desinfektion verunreinigter Teile

D für Trinkwasser-Installation ${ }^{\text {a }}$

D1 Austausch, Abtrennung oder Reparatur defekter Teile

D2 Mechanische/chemische Reinigung und/oder Desinfektion verunreinigter Teile

SSicherungsmaßnahmen, um Zutritt durch Unbefugte zu verhindern

S1 Sicherungsmaßnahmen, um Zutritt durch Unbefugte zu verhindern

OSonstiges

$01 \quad$ Sonstiges

ENotfallmaßnahmen im Sinne der Verbrauchergesundheit und -sicherheit

E1 Benachrichtigung der Verbraucher und Anweisungen (z. B. Entnahmeverbot, Abkochgebot, vorübergehende Gebrauchseinschränkung)

E2 Vorübergehende Bereitstellung einer alternativen Trinkwasserversorgung (z. B. Wasser in Flaschen, Behältern, Tankwagen)

aEinschließlich häuslicher Trinkwasser-Installationen, aus denen Wasser an die Öffentlichkeit abgegeben wird.

\section{Tab. B10 Codes für den Zeitrahmen der Abhilfemaßnahmen}

Code

I

$\mathrm{S}$

M

L

\section{Zeitrahmen}

Sehr kurzfristig, d. h. nicht mehr als 1 Tag

Kurzfristig, d. h. nicht mehr als 30 Tage

Mittelfristig, d. h. mehr als 30 Tage, aber nicht mehr als 1 Jahr

Langfristig, d. h. mehr als 1 Jahr

Tab. B11 Kommunikation der im Land verfügbaren Informationen über die Qualität von Trinkwasser. (Angaben hier fakultativ, da in der Berichterstattung nach TW-RL ausreichend berücksichtigt)

Land

Jahr

Medium Ja/Nein $\quad$ Ort der Information ${ }^{\text {a }}$

11.1 Webseite

11.2 Jahresbericht (Land)

11.3 Jahresbericht (regional)

11.4 Informationsblätter

11.5 Rundschreiben

11.6 Wasserrechnungen ${ }^{b}$

11.7 Öffentliche Veranstaltungen

11.8 Lokalzeitungen

11.9 Öffentlich zugängliche Akten ${ }^{c}$

11.10 Sonstiges

aBei dem Ort sollte es sich um die Stelle handeln, an die sich das BMG/UBA wenden kann, um die jeweils aufgeführten Arten von Informationen zu erhalten. ${ }^{\text {Informationen }}$ zur Qualität von Trinkwasser in den Wasserrechnungen an die Kunden. 'Hier sind Akten gemeint, die vom Wasserversorger, dem Gemeinderat oder der Regierungsbehörde bzw. dem Ministerium geführt und von jedermann während der Öffnungszeiten eingesehen werden können. 
Tab. B12 Art der im Land öffentlich zugänglichen Informationen. (Angaben hier fakultativ, wenn in der Berichterstattung nach TW-RL ausreichend berücksichtigt)

Land

Jahr

Art der verfügbaren Informationen

Ja/Nein

Ort der Information ${ }^{\text {a }}$

12.1 Landesweite Zusammenfassung über die Einhaltung der TrinkwV 2001 in den WVG

12.2 Regionale Zusammenfassung über die Einhaltung der TrinkwV 2001 in den WVG

12.3 Zusammenfassung der Einhaltung der TrinkwV 2001, bezogen auf das WVG

12.4 Einzelne Überwachungsergebnisse für das WVG

12.5 Überwachungsergebnisse für sehr kleine WVG (in denen im

Durchschnitt weniger als $10 \mathrm{~m}^{3} /$ Tag verteilt und weniger als

50 Personen versorgt werden)

12.6 Informationen über Einhaltung strengerer nationaler Standards

12.7 Informationen über Einhaltung zusätzlicher nationaler Standards

12.8 Informationen auf der Ebene eines WVG über Nichteinhaltungen

12.9 Informationen über die Trinkwasserressource( $\mathrm{n}$ ) im betreffenden WVG

12.10 Sonstiges

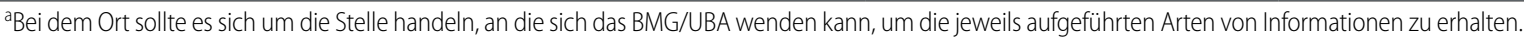

Anhang zu Tabelle A1 bis A12 und Tabelle B1 bis B12 (informativ)

\begin{tabular}{|c|c|c|c|c|c|c|}
\hline \multirow[t]{2}{*}{ Art der Information } & \multirow[t]{2}{*}{$\begin{array}{l}\text { Ggf. Bezug } \\
\text { TrinkwV } 2001\end{array}$} & \multirow[t]{2}{*}{$\begin{array}{l}\text { Bezug } \\
\text { Tabelle }\end{array}$} & \multicolumn{3}{|c|}{$\begin{array}{l}\text { Art der nach TW-RL oder TrinkwV } \\
2001 \text { betroffenen Parameter }\end{array}$} & \multirow[t]{2}{*}{$\begin{array}{l}\text { Periodizität/ } \\
\text { Fristen }\end{array}$} \\
\hline & & & $M$ & C & I & \\
\hline $\begin{array}{l}\text { Allgemeine Informationen zu Rahmenbedin- } \\
\text { gungen der Trinkwasserversorgung }\end{array}$ & & $\begin{array}{l}\text { - Tab. } \mathbf{A} 1 \text { oder } \\
\text { - Tab. B1 }\end{array}$ & & & & Jährlich \\
\hline $\begin{array}{l}\text { Jahresübersicht zur Einhaltung der Überwa- } \\
\text { chungshäufigkeit im WVG }\end{array}$ & $\begin{array}{l}\text { § } 14 \text { Abs. } 1 \\
\text { Anlage } 4 \text { Teil I } \\
\text { Abs. } 1 \text { und } 2 \\
\text { Anlage 4 Teil II }\end{array}$ & $\begin{array}{l}\text { - Tab. A4 oder } \\
\text { - Tab. B4 }\end{array}$ & + & + & + & Jährlich \\
\hline $\begin{array}{l}\text { Landesweite zusammenfassende Informatio- } \\
\text { nen zur Qualität von Trinkwasser in WVG }\end{array}$ & & $\begin{array}{l}\text { - Tab. A5a oder } \\
\text { - Tab. B5a } \\
\text { - Tab. A5b oder } \\
\text { - Tab. B5b }\end{array}$ & + & + & + & Jährlich \\
\hline $\begin{array}{l}\text { Informationen zu Nichteinhaltungen der Para- } \\
\text { meterwerte bei Trinkwasser in WVG }\end{array}$ & & $\begin{array}{l}\text { - Tab. A6 oder } \\
\text { - Tab. B6 }\end{array}$ & + & + & + & Jährlich \\
\hline $\begin{array}{l}\text { Kommunikation der verfügbaren Informatio- } \\
\text { nen über die Qualität von Trinkwasser }\end{array}$ & $\S 21$ Abs. 1 & $\begin{array}{l}\text { Tab. A11, ggf. } \\
\text { - Tab. B11 }\end{array}$ & & & & Jährlich \\
\hline $\begin{array}{l}\text { Art der Informationen, die der Öffentlichkeit zur } \\
\text { Verfügung stehen }\end{array}$ & $\S 21$ Abs. 1 & $\begin{array}{l}\text { - Tab. A12, ggf. } \\
\text { - Tab. B12 }\end{array}$ & & & & Jährlich \\
\hline
\end{tabular}

${ }^{\mathrm{a}} \mathrm{M}$ mikrobiologische Parameter, $\mathrm{C}$ chemische Parameter, I Indikatorparameter. 


\section{Informationen, die nach Artikel 9 TW-RL in Verbindung mit § 10 TrinkwV 2001 über zugelassene Abweichungen verlangt werden}

Die Informationen über eine bestehende zugelassene Abweichung nach Artikel 9 TW-RL und $\$ 10$ TrinkwV 2001 sind dem UBA unter Beachtung der in Artikel 9 TW-RL bzw. $\$ 10$ TrinkwV 2001 genannten Vorgaben zuzuleiten. Analysenergebnisse im Rahmen einer zugelassenen Abweichung stellen eine Überschreitung dar, über die auch nach Artikel 13 TWRL (bzw. $\$ 21$ Absatz 2 TrinkwV 2001) zu berichten ist, wenn die Abweichung in einem WVG zugelassen worden ist, in dem mehr als $1000 \mathrm{~m}^{3}$ Trinkwasser pro Tag geliefert oder mehr als 5000 Personen versorgt werden (bzw. wenn die Abweichung in einem WVG mit dem TrinkwVspezifischen Größenbereich zugelassen worden ist, s. hierzu unter 3.1).

Nach TW-RL (und TrinkwV 2001) ist nur über Abweichungen von einem Parameterwert (Grenzwert) der chemischen Parameter gemäß Anhang I Teil B TWRL (Anlage 2 TrinkwV 2001) zu berichten.

Vorlagen für die Meldungen nach Artikel 9 TW-RL bilden die Formblätter D1, D2 und D3. Sie berücksichtigen auch die Anforderungen an die Meldungen nach $\$ 10$ TrinkwV 2001.

Die einzelne Meldung ist als XMLDatei über die für Trinkwasser spezifizierte XML-Schnittstelle in die zentrale Datenhaltung des „WasserBLIcK“ bei der BfG einzustellen.

\subsection{Formblatt D1}

Betrifft Informationen zu einer ersten zugelassenen Abweichung gemäß den Anforderungen von Artikel 9 Absatz 1 Satz 1 und 2 in Verbindung mit Absatz 7 TWRL und $\$ 10$ Absatz 2 und 3 TrinkwV 2001. Es ist bei Abweichungen in Bezug auf ein WVG, in dem mehr als $1000 \mathrm{~m}^{3}$ Trinkwasser pro Tag geliefert oder mehr als 5000 Personen versorgt werden, dem UBA innerhalb von 6 Wochen nach der Zulassung vorzulegen, sodass das BMG in die Lage versetzt wird, die EU-Kommission gemäß Artikel 9 Absatz 7 TW-RL über die zugelassene Abweichung innerhalb von 2 Monaten zu unterrichten.

\subsection{Formblatt D2}

Dient der Meldung über eine zweite zugelassene Abweichung gemäß den Anforderungen von Artikel 9 Absatz 1 Satz 3 TW-RL in Verbindung mit $\$ 10$ Absatz 5 TrinkwV 2001. Es ist dem UBA innerhalb von 6 Wochen nach der Zulassung vorzulegen, sodass das BMG in der Lage ist, die EU-Kommission gemäß Artikel 9 Absatz 1 TW-RL über die zugelassene Abweichung innerhalb von 2 Monaten zu unterrichten.

\subsection{Formblatt D3}

Enthält die Informationen, die das BMG in die Lage versetzen, ggf. einen Antrag auf Zulassung einer dritten Abweichung nach Artikel 9 Absatz 2 TW-RL bei der EU-Kommission zu stellen. Formblatt D3 ist gemäß den Anforderungen von Artikel 9 Absatz 2 TW-RL in Verbindung mit $\$ 10$ Absatz 6 TrinkwV 2001 spätestens 5 Monate vor dem Zeitpunkt zu übersenden, zu dem die zugelassene Abweichung erforderlich wird. 
Formblatt D1 Meldung über die Zulassung einer Abweichung nach Artikel 9 Absatz 7 TW-RL in Verbindung mit § 10 Absatz 2 und 3 TrinkwV 2001. (Erste zugelassene Abweichung) ${ }^{\mathrm{a}}$

Land

D1.1 Aktenzeichen der zuständigen obersten Landesbehörde für die Zulassung der Abweichung

D1.2 Bezeichnung des Wasserversorgungsgebiets

ID

NUTS

D1.4 Gesamtvolumen des gelieferten Wassers in $\mathrm{m}^{3} / \mathrm{Tag}$

D1.5 Bevölkerungsstärke des Wasserversorgungsgebiets

D1.6 Zahl der von der zugelassenen Abweichung betroffenen Bevölkerung ${ }^{b}$

D1.7 Sind relevante Lebensmittelbetriebe betroffen? (Ja oder Nein)

D1.8 Betroffener Parameter

D1.9 Für die zugelassene Abweichung festgelegter Höchstwert (einschl. Einheit) $^{c}$

D1.10 Zusammenfassung der vorherigen diesbezüglichen Überwachungsergebnisse

D1.10.1 Anzahl der Untersuchungen

D1.10.2 Vorherige diesbezügliche Überwachungsergebnisse über einen Zeitraum von höchstens 3 Jahren (min./med./max.) $)^{c}$

D1.11 Gründe für die Zulassung der Abweichung ${ }^{d}$

D1.12 Erläuterung der vorgeschlagenen Abhilfemaßnahme, einschl. Zeitplan ${ }^{\mathrm{e}}$

D1.13 Vorgeschlagene Überwachungsmaßnahmen

D1.14 Anfangsdatum der ersten zugelassenen Abweichung

D1.15 Enddatum der ersten zugelassenen Abweichung

D1.16 Kontaktstelle im Land

D1.16.1 Zuständige Behörde

D1.16.2 Ansprechpartner

D1.16.3 Anschrift

D1.16.4 Telefon

D1.16.5 Fax

D1.16.6 E-Mail

${ }^{a}$ Die zuständige oberste Landesbehörde unterrichtet das Umweltbundesamt unter Verwendung dieses Formblattes über jede Zulassung einer Abweichung in einem WVG, in dem mehr als $1000 \mathrm{~m}^{3}$ Trinkwasser pro Tag geliefert oder mehr als 5000 Personen versorgt werden, spätestens 6 Wochen nach Zulassung. bFalls nicht mit D1.5 identisch. Wenn nicht bekannt, Schätzwert angeben. 'Einheiten nach TrinkwV 2001. 'Ausführlich in Textform begründen unter Verwendung eines oder mehrerer Codes für die Ursachen einer Nichteinhaltung aus Anlage 4-1. eHier werden aus Anlage 4-1 die Codes für Abhilfemaßnahmen (es können mehrere Codes zutreffen) und für den Zeitrahmen einer Abhilfemaßnahme verwendet. Bei mehr als einer Abhilfemaßnahme wird ihr der jeweilige Zeitrahmen zugeordnet 


\section{Bekanntmachungen - Amtliche Mitteilungen}

Formblatt D2 Meldung über die Zulassung einer Abweichung nach Artikel 9 Absatz 1 TW-RL in Verbindung mit § 10 Absatz 5 TrinkwV 2001. (Zweite zugelassene Abweichung) $^{2}$

Land

D2.1 Aktenzeichen der zuständigen obersten Landesbehörde für die Zulassung der Abweichung

D2.2 Bezeichnung des Wasserversorgungsgebiets

D2.3 Code des Wasserversorgungsgebiets NUTS

D2.4 Gesamtvolumen des gelieferten Wassers in $\mathrm{m}^{3} / \mathrm{Tag}$

D2.5 Bevölkerungsstärke des Wasserversorgungsgebiets

D2.6 Zahl der von der zugelassenen Abweichung betroffenen Bevölkerung ${ }^{b}$

D2.7 Sind relevante Lebensmittelbetriebe betroffen (Ja oder Nein)

D2.8 Betroffener Parameter

D2.9 Für die zugelassene Abweichung festgelegter Höchstwert (einschl. Einheit)c

D2.10 Datum der Prüfung der ersten zugelassenen Abweichung

D2.11 Schlussfolgerungen der Prüfung der ersten zugelassenen Abweichung

D2.12 Zusammenfassung der Überwachungsergebnisse während der Dauer der ersten zugelassenen Abweichung

D2.12.1 AZ der zuständigen obersten Landesbehörde für die Zulassung der ersten Abweichung $^{\mathrm{d}}$

D2.12.2 Anzahl der Untersuchungen

D2.12.3 Überwachungsergebnisse während der Dauer der ersten Zulassung (min./med./max.) $)^{c}$

D2.13 Gründe für die Zulassung der zweiten Abweichunge

D2.14 Erläuterung der vorgeschlagenen Abhilfemaßnahme, einschl. Zeitplan ${ }^{f}$

D2.15 Vorgeschlagene Überwachungsmaßnahmen

D2.16 Anfangsdatum der zweiten zugelassenen Abweichung

D2.17 Enddatum der zweiten zugelassenen Abweichung

D2.18 Kontaktstelle im Land

D2.18.1 Zuständige Behörde

D2.18.2 Ansprechpartner

D2.18.3 Anschrift

D2.18.4 Telefon

D2.18.5 Fax

D2.18.6 E-Mail

aDie zuständige oberste Landesbehörde unterrichtet das Umweltbundesamt unter Verwendung dieses Formblattes über jede Zulassung einer Abweichung in einem WVG, in dem mindestens $10 \mathrm{~m}^{3}$ Trinkwasser pro Tag geliefert oder mindestens 50 Personen versorgt werden oder das Wasser im Rahmen einer öffentlichen oder gewerblichen Tätigkeit verwendet wird, spätestens 6 Wochen nach Zulassung. ${ }^{b}$ Falls nicht mit D2.5 identisch. Wenn nicht bekannt, Schätzwert angeben. ${ }^{\mathrm{c} E i n h e i t e n ~ n a c h ~ T r i n k w V ~ 2001 . ~}{ }^{\mathrm{d}}$ Eine Kopie des maßgeblichen Formblattes D1 kann zu Informationszwecken beigefügt werden. ${ }^{e}$ Ausführlich in Textform begründen unter Verwendung eines oder mehrerer Codes für die Ursachen einer Nichteinhaltung aus Anlage 4-1. 'Hier werden aus Anlage 4-1 die Codes für Abhilfemaßnahmen (es können mehrere Codes zutreffen) und für den Zeitrahmen einer Abhilfemaßnahme verwendet. Bei mehr als einer Abhilfemaßnahme wird ihr der jeweilige Zeitplan zugeordnet. 
Formblatt D3 Antrag auf Zulassung einer dritten Abweichung nach Artikel 9 Absatz 2 TW-RL in Verbindung mit $§ 10$ Absatz 6 TrinkwV 2001 $^{\mathrm{a}}$

\section{Land}

D3.1 AZ der zuständigen obersten Landesbehörde für

die Zulassung der Abweichung

D3.2 Bezeichnung des Wasserversorgungsgebiets

\begin{tabular}{|ll}
\hline D3.3 & Code des Wasserversorgungsgebiets \\
\hline D3.4 & Gesamtvolumen des verteilten Wassers in $\mathrm{m}^{3} / \mathrm{Tag}$ \\
\hline
\end{tabular}

D3.5 Bevölkerungsstärke des Wasserversorgungsgebiets

D3.6 Anzahl der von der zugelassenen Abweichung betroffenen Bevölkerung ${ }^{\text {b }}$

D3.7 Sind relevante Lebensmittelbetriebe betroffen (Ja oder Nein)

D3.8 Betroffener Parameter

D3.9 Für die zugelassene Abweichung festgelegter Höchstwert (einschl. Einheit) ${ }^{c}$

D3.10 Datum der Prüfung der zweiten zugelassenen Abweichung

D3.11 Schlussfolgerungen der Prüfung der zweiten zugelassenen Abweichung

D3.12 Zusammenfassung der Überwachungsergebnisse während der Dauer der zweiten zugelassenen Abweichung

D3.12.1 AZ der zuständigen obersten Landesbehörde für die Zulassung der ersten Abweichung ${ }^{d}$

D3.12.2 AZ der zuständigen obersten Landesbehörde für die Zulassung der zweiten Abweichung $^{d}$

D3.12.3 Anzahl der Untersuchungen

D3.12.4 Überwachungsergebnisse während der Dauer der zweiten Zulassung (min./med./max.) $)^{c}$

D3.13 Gründe für die Zulassung der dritten Abweichung ${ }^{\mathrm{e}}$

D3.14 Erläuterung der vorgeschlagenen Abhilfemaßnahme, einschl.Zeitplan ${ }^{f}$

D3.15 Vorgeschlagene Überwachungsmaßnahmen

D3.16 Anfangsdatum der dritten Abweichung

D3.17 Enddatum der dritten Abweichung

D3.18 Kontaktstelle im Land

D3.18.1 Zuständige Behörde

D3.18.2 Ansprechpartner

D3.18.3 Anschrift

D3.18.4 Telefon

D3.18.5 Fax

D3.18.6 E-Mail

aDie zuständige oberste Landesbehörde übersendet dem Umweltbundesamt Formblatt D3 mit dem Antrag auf Zulassung einer dritten Abweichung in Bezug auf ein WVG, in dem mindestens $10 \mathrm{~m}^{3}$ Trinkwasser pro Tag geliefert oder mindestens 50 Personen versorgt werden oder das Wasser im Rahmen einer öffentlichen oder gewerblichen Tätigkeit verwendet wird, spätestens 5 Monate vor Ablauf der zweiten Abweichungsfrist. bFalls nicht mit D3.5 identisch. Wenn nicht bekannt, Schätzwert angeben. 'Einhei-

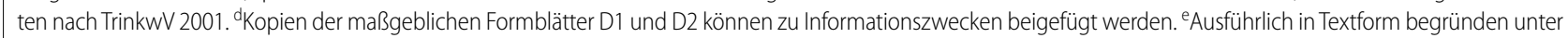
Verwendung eines oder mehrerer Codes für die Ursachen einer Nichteinhaltung aus Anlage 4-1. fHier werden aus Anlage 4-1 die Codes für Abhilfemaßnahmen (es können mehrere Codes zutreffen) und für den Zeitrahmen einer Abhilfemaßnahme verwendet. Bei mehr als einer Abhilfemaßnahme wird ihr der jeweilige Zeitplan zugeordnet. 


\section{Anlage 4-1 Codes für die \\ Formblätter D1, D2 und D3}

Codes für die Ursachen einer Nichteinhaltung (zu D1, D2 und D3)

Code Ursache

C Auf Einzugsgebiet zurückzuführen

T AufWasserwerk zurückzuführen

$\mathrm{P} \quad$ Auf öffentliches Verteilungsnetz zurückzuführen

D Auf häusliche Trinkwasser-Installation zurückzuführen

O Andere Ursache

$\mathrm{S} \quad$ Kombination an Ursachen

U Unbekannte Ursache

\section{Codes für Abhilfemaßnahmen bei Nichteinhaltung (zu D1, D2 und D3)}

\section{Code}

Cfür Einzugsgebiet

C1

$\mathrm{C} 2$

Tfür Aufbereitung

$\mathrm{T} 1$

Pfür öffentliches Verteilungsnetz

P1

P2

D für Trinkwasser-Installation ${ }^{\mathrm{a}}$

D1

D2

SSicherungsmaßnahmen, um Zutritt durch Unbefugte zu verhindern

$\mathrm{S} 1$

OSonstiges

01

ENotfallmaßnahmen im Sinne der Verbrauchergesundheit und -sicherheit

E1

E2

aEinschließlich häuslicher Trinkwasser-Installationen, aus denen im Rahmen einer gewerblichen oder öffentlichen Tätigkeit Trinkwasser abgegeben wird.

Beschreibung der Abhilfemaßnahme

Maßnahme(n) zur Behebung bzw. Minderung der Ursache

Maßnahme(n) zum Ersatz der Wasserressource

Einführung, Aufrüstung oder Ertüchtigung der Aufbereitung

Austausch, Abtrennung oder Reparatur defekter Teile reinigter Teile

Austausch, Abtrennung oder Reparatur defekter Teile

Mechanische/chemische Reinigung und/oder Desinfektion verunreinigter Teile

Sonstiges kung) sorgung (z. B. Wasser in Flaschen, Behältern, Tankwagen)

Mechanische/chemische Reinigung und/oder Desinfektion verun-

Sicherungsmaßnahmen, um Zutritt durch Unbefugte zu verhindern

Benachrichtigung der Verbraucher und Anweisungen (z. B. Entnahmeverbot, Abkochgebot, vorübergehende Gebrauchseinschrän-

Vorübergehende Bereitstellung einer alternativen Trinkwasserver-

Codes für den Zeitrahmen der Abhilfemaßnahmen (zu D1, D2 und D3)

Code

I Sehr kurzfristig, d. h. nicht mehr als 1 Tag

S Kurzfristig, d. h. nicht mehr als 30 Tage

M Mittelfristig, d. h. mehr als 30 Tage, aber nicht mehr als 1 Jahr

L Langfristig, d. h. mehrals 1 Jahr 


\section{Anlage 4-2 (informativ)}

Zusammenfassung der Anforderungen an die Meldungen nach Artikel 9 TW-RL bzW. $\$ 10$ TrinkwV 2001 (zugelassene Abweichungen) durch die zuständigen obersten Landesbehörden

\begin{tabular}{|lllll}
\hline Art der Information & $\begin{array}{l}\text { Bezug } \\
\text { TrinkwV 2001 }\end{array}$ & $\begin{array}{l}\text { Ggf. Bezug } \\
\text { TW-RL }\end{array}$ & $\begin{array}{l}\text { Bezug } \\
\text { Formblatt }\end{array}$ & Periodizität/Fristen \\
\hline $\begin{array}{l}\text { Informationen über die Zulas- } \\
\text { sung der ersten Abweichung }\end{array}$ & $\S 10$ Abs. 2 und Abs. 3 & Art. 9 Abs. 7 & D1 & $\begin{array}{l}\text { Spätestens 6Wochen nach } \\
\text { Zulassung }\end{array}$ \\
\hline $\begin{array}{l}\text { Informationen über die Zulas- } \\
\text { sung der zweiten Abweichung }\end{array}$ & $\S 10$ Abs. 5 & Art. 9 Abs. 1 & D2 & $\begin{array}{l}\text { Spätestens 6Wochen nach } \\
\text { Zulassung }\end{array}$ \\
\hline $\begin{array}{l}\text { Antrag auf Zulassung der drit- } \\
\text { ten Abweichung }\end{array}$ & $\S 10$ Abs. 6 & Art. 9 Abs. 2 & D3 & $\begin{array}{l}\text { Spätestens 5 Monate vor Ablauf } \\
\text { der zweiten zugelassenen } \\
\text { Abweichung }\end{array}$ \\
\hline
\end{tabular}

